\title{
Primordial black holes as a novel probe of primordial gravitational waves. II: Detailed analysis
}

\author{
Tomohiro Nakama ${ }^{1}$ and Teruaki Suyama ${ }^{2}$ \\ ${ }^{1}$ Department of Physics and Astronomy, Johns Hopkins University \\ 3400 N. Charles Street, Baltimore, Maryland 21218, USA \\ ${ }^{2}$ Research Center for the Early Universe (RESCEU), \\ Graduate School of Science, The University of Tokyo, \\ Bunkyo-ku, Tokyo 113-0033, Japan
}

(Dated: September 13, 2018)

\begin{abstract}
Recently we have proposed a novel method to probe primordial gravitational waves from upper bounds on the abundance of primordial black holes (PBHs). When the amplitude of primordial tensor perturbations generated in the early Universe is fairly large, they induce substantial scalar perturbations due to their second-order effects. If these induced scalar perturbations are too large when they reenter the horizon, then PBHs are overproduced, their abundance exceeding observational upper limits. That is, primordial tensor perturbations on superhorizon scales can be constrained from the absence of PBHs. In our recent paper we have only shown simple estimations of these new constraints, and hence in this paper, we present detailed derivations, solving the Einstein equations for scalar perturbations induced at second order in tensor perturbations. We also derive an approximate formula for the probability density function of induced density perturbations, necessary to relate the abundance of PBHs to the primordial tensor power spectrum, assuming primordial tensor perturbations follow Gaussian distributions. Our new upper bounds from PBHs are compared with other existing bounds obtained from big bang nucleosynthesis, cosmic microwave background, LIGO/Virgo and pulsar timing arrays.
\end{abstract}

PACS numbers: 


\section{INTRODUCTION}

A stochastic background of primordial gravitational waves (PGWs) with a huge range of wavelengths may have been generated in the early Universe. Their power spectrum reflects physical conditions in the early Universe, and hence its constraints provide valuable information for cosmology. PGWs of largest observable wavelengths have been constrained by Planck [1] and BICEP2 [2], while those of shorter wavelengths have been constrained by limits on $N_{\text {eff }}$, the effective number of degrees of freedom of relativistic fermions, at big bang nucleosynthesis (BBN) through the current abundance of the light elements [3], or at photon decoupling through the anisotropy of cosmic microwave background (CMB) [4, [5]. Recently PGWs on smaller scales have been constrained by upper limits on the deviation of the CMB photons' energy spectrum from the Planck distribution [6, 7]. Though BBN and CMB constrain PGWs of a wide range of wavelengths, these upper bounds, obtained through $N_{\text {eff }}$, entail an assumption about the number of relativistic species in the early Universe, as is discussed later. Furthermore, to obtain BBN or CMB bounds we implicitly assume that any physical mechanisms, both known and unknown, increase $N_{\text {eff }}$, from the standard value $N_{\text {eff }}=3.046$ [8]. However, $N_{\text {eff }}$ can decrease e.g. in brane world scenarios [9 11]. Recently we proposed a new method to constrain PGWs in our recent work [12], which is also applicable on a wide range of wavelengths and in addition does not depend on the aforementioned assumptions much. In this paper, we present detailed derivations of the results presented there.

Our new method uses the formation of primordial black holes (PBHs), formed in the early Universe, well before the cosmic structure formation. One of the simple and plausible mechanisms to form PBHs is the direct collapse of density fluctuations during the radiation-dominated era, which happens when the fractional density perturbation of order unity reenters the Hubble horizon 13 15]. See also [16] for an updated discussion of the formation condition and 17 23] for numerical simulations of the PBH formation process. There is no conclusive evidence for the existence of PBHs in the present as well as in the past and upper bounds on their abundance over a wide mass range have been obtained by various methods (see e.g. [24] and references therein). One of the cosmological implications of their absencesis to constrain the power spectrum of the curvature perturbation $[25,26]^{\dagger 1}$. In a broader context, $\mathrm{PBHs}$ provide valuable information to exclude models of the early Universe which predict an overproduction of PBHs.

As we have briefly discussed in our recent work [12], PBHs can also be used to constrain tensor perturbations generated in the early Universe, exiting the horizon once and reentering the horizon later. This is because largetensor perturbations induce large scalar perturbations (induced scalar perturbations) at second order in tensor perturbations. If primordial tensor perturbations are too large, induced scalar perturbations become also too large, and then they collapse to overproduce PBHs shortly after their horizon reenty, exceeding existing upper limits. That is, primordial tensor perturbations can be constrained from upper limits on $\mathrm{PBHs}^{\dagger 2 \dagger 3}$. Whereas we have presented only

$\dagger 1$ Other methods to constrain primordial scalar perturbations on small scales include CMB spectral distortions [27 36], acoustic reheating [37, 38] and ultracompact minihalos [39, 40].

$\dagger^{2}$ Second-order effects of scalar perturbations to induce tensor perturbations (termed induced gravitational waves) have been discussed in the literature [41 47]; we can place upper bounds on scalar perturbations(, which can be translated into upper bounds on the abundance of PBHs [48 50],) from the non-detection of GWs. Note that our present paper discusses an effect opposite to this generation of induced gravitational waves.

$\dagger 3$ The direct gravitational collapse of nonlinear localized gravitational waves has been discussed in the liter- 
simple estimations to obtain these new constraints in [12], in the present paper we show detailed derivations for them.

Due to our ignorance of the physics in the early Universe, new upper limits on tensor perturbations on small scales in themselves would be worthwhile. In addition, there are models of the early Universe [60 67] which can predict large tensor perturbations on small scales, which makes our new upper limits even more valuable (see the next section). Note that, if a model predicts large tensor perturbations on small scales but also large scalar perturbations at the same time, then such a model would be more severely constrained from the absence of PBHs generated from the first-order scalar perturbations. In this paper we consider PBH formation only from induced scalar perturbations, second order in tensor perturbations, and thus our bounds on tensor perturbations are conservative or model-independent, in the sense that these bounds do not depend onfirst-order scalar perturbations on small scales. Importantly, there are models of the early Universe which predict not only large tensor perturbations, but also large tensor-to-scalar ratio on small scales, and our PBH bounds are particularly useful to constrain these types of models, some of which are reviewed in the next section.

This paper is organized as follows; In Sec. II we review some of the early Universe models 60 67] which predict large tensor-to-scalar ratio on small scales. In Sec. III the radiation density perturbation generated from tensor perturbations is calculated. Section IV is dedicated to a discussion of upper bounds on tensor modes from PBHs along with a comparison with those obtained from other methods, and we conclude in Sec. V.

\section{EARLY UNIVERSE MODELS PREDICTING LARGE TENSOR-TO-SCALAR RATIO ON SMALL SCALES}

In [60], tensor power spectra were shown to be blue (i.e. larger power on smaller scales) in cyclic/ekpyrotic models, with the spectrum of scalar perturbations kept slightly red (smaller power on smaller scales) to match observations on large scales. The cyclic Universe entails the periodic collisions of orbifold planes moving in an extra spatial dimension, which is equivalently described by a scalar field rolling back and forth in an effective potential. Each cycle consists of an accelerated expansion phase, a slow contraction phase (the ekpyrotic phase), during which the Universe is dominated by the kinetic energy as well as the negative potential energy of the scalar field and primordial fluctuations are generated, a rapid contraction phase followed by a bounce at which matter and radiation are generated, a phase dominated by the kinetic energy of the scalar field, a radiation-dominated, expanding phase, and finally a phase dominated by matter and dark energy. The spectrum of scalar perturbations can be adjusted to be slightly red by tuning the scalar field potential during the ekpyrotic phase, and the tensor spectrum turns out to be blue up to the scale corresponding to the end of the ekpyrotic phase. For early Universe scenarios where the spectrum of tensor perturbations is strongly blue, probing them on CMB scales may be challenging, while constraints on small-scale components, such as those discussed in this paper, may provide useful information. Indeed, they noted that the strongest constraint on their model parameters is obtained

ature [51 59] and so tensor perturbations may also be constrained using this phenomenon. Still, the initial conditions and dynamics of cosmological nonlinear gravitational waves during the radiation-dominated era have not been well understood. Since the dynamics of nonlinear radiation density perturbations is better understood, we consider only scalar perturbations induced by the tensor perturbation. 
from BBN constraints on high-frequency PGWs.

If the inflaton violates the null energy condition (NEC, $\rho+p \geq 0$ ), the Hubble parameter increases during inflation (super inflation) and the spectral tilt $n_{T}$ becomes positive, since $n_{T}=$ $-2 \epsilon \equiv 2 \dot{H} / H^{2} \propto-(\rho+p)$. In [61] it was shown that NEC can be violated without the instability of fluctuations of the inflaton. There a toy model was introduced, with the energy density of the NEC-violating inflaton $\rho=-\dot{\phi}^{2} / 2+V_{0} e^{-\lambda \phi / M_{\mathrm{pl}}}$, which leads to a stage of pole-like inflation, when $a(t) \sim(-t)^{p}, t<0, p=-2 / \lambda^{2}<0$. The background and fluctuations are shown to be stable at the classical level. It was noted that in this model, some mechanism, quantum effects or another field, is necessary to avoid singularity at $t \rightarrow 0$ and to drive the Universe into a radiation-dominated epoch.

The spectrum of tensor perturbations generated during a super inflation in the framework of loop quantum cosmology (LQC) is calculated in [62]. There a strong blue tile with $n_{T} \simeq 2$ was obtained, while the form of the inflaton potential to realize a scale-invariant power spectrum of scalar perturbations was also discussed in their previous works. In their scenario, the nondimensional power spectrum of tensor perturbations on smallest scales is roughly given by the square of the Hubble parameter $H_{\mathrm{e}}$ at the end of inflation in units of the Planck scale, and this implies that $H_{\mathrm{e}}$ can be constrained e.g. by our PBH constraints. They note that $H_{\mathrm{e}}$ is, in principle, also related to the amplitude of scale-invariant curvature perturbations as well, but such a relation has not been obtained yet in the scenarios they consider.

Large tensor perturbations on small scales may also be realized in the framework of the so-called generalized G-inflation ( $G^{2}$-inflation) [63]. The action of $G^{2}$-inflation contains four generic functions $K, G_{3}, G_{4}, G_{5}$ of $\phi$ and $X=-\partial_{\mu} \phi \partial^{\mu} \phi / 2$. The quadratic action for the tensor perturbations is

$$
\begin{gathered}
S_{T}^{(2)}=\frac{1}{8} \int d t d^{3} x a^{3}\left[\mathcal{G}_{T} \dot{h}_{i j}^{2}-\frac{\mathcal{F}_{T}}{a^{2}}\left(\nabla h_{i j}\right)^{2}\right], \\
\mathcal{G}_{T} \equiv 2\left[G_{4}-2 X G_{4 X}-X\left(H \dot{\phi} G_{5 X}-G_{5 \phi}\right)\right], \quad \mathcal{F}_{T} \equiv 2\left[G_{4}-X\left(\ddot{\phi} G_{5 X}+G_{5 \phi}\right)\right] .
\end{gathered}
$$

The squared sound speed is $c_{T}^{2}=\mathcal{F}_{T} / \mathcal{G}_{T}$, which is not necessarily unity in general cases. The parameters $\epsilon \equiv-\dot{H} / H^{2}, f_{T} \equiv \dot{\mathcal{F}}_{T} / H \mathcal{F}_{T}$ and $g_{T} \equiv \dot{\mathcal{G}_{T}} / H \mathcal{G}_{T}$ are introduced and they are assumed to be nearly constant. The nondimensional power spectrum of the tensor perturbations was obtained as

$$
\mathcal{P}_{T}=\left.8 \gamma_{T} \frac{\mathcal{G}_{T}^{1 / 2}}{\mathcal{F}_{T}^{3 / 2}} \frac{H^{2}}{4 \pi^{2}}\right|_{-k y_{T}=1},
$$

where

$$
\nu_{T} \equiv \frac{3-\epsilon+g_{T}}{2-2 \epsilon-f_{T}+g_{T}}, \quad \gamma_{T}=2^{2 \nu_{T}-3}\left|\frac{\Gamma\left(\nu_{T}\right)}{\Gamma(3 / 2)}\right|^{2}\left(1-\epsilon-\frac{f_{T}}{2}+\frac{g_{T}}{2}\right), \quad d y_{T} \equiv \frac{c_{T}}{a} d t .
$$

The tensor spectral tilt is given by $n_{T}=3-2 \nu_{T}$, and the tensor spectrum is blue $\left(0<n_{T}\right)$ if $4 \epsilon+3 f_{T}-g_{T}<0$. Also, if the sound speed becomes temporarily small, tensor perturbations are enhanced on the corresponding scales.

A slightly red spectrum of the curvature perturbation, while keeping the tensor spectrum strongly blue-tilted, was also shown to be realized during a stringy thermal contracting phase at temperatures beyond the so-called Hagedorn temperature (the Hagedorn phase) in [64], assuming a nonsingular bounce. In that scenario, primordial curvature perturbations originate from statistical thermal fluctuations, not by scalar field quantum fluctuations.

Scalar and tensor perturbations in large field chaotic models with non-Bunch-Davies (non$\mathrm{BD})$ initial states were analyzed in [65], and it was shown that in that model also gravitational 
waves can be blue while maintaining slightly red scalar perturbations. Normally, initial states for perturbations are chosen to be Bunch-Davies (BD) vacuum states, namely, perturbation modes on sub-Hubble scales effectively propagate in vacuum states associated with flat space. NonBD initial states were characterized by the Bogoliubov coefficients for each $k$ mode and for both scalar and tensor perturbations, which were denoted by $\alpha_{k}^{S}, \beta_{k}^{S}, \alpha_{k}^{T}, \beta_{k}^{T}$, with $\left(\alpha_{k}^{S, T}, \beta_{k}^{S, T}\right)=(1,0)$ corresponding to the standard BD initial states. These parameters are determined by unknown high energy physics, and depending on the choice of the above parameters, blue gravitational waves were obtained while maintaining the scalar perturbations slightly red.

Blue gravitational waves with slightly red scalar perturbations were also obtained without violating NEC by breaking the spatial diffeomorphism, usually imposed on the dynamics of perturbations, in the context of effective theory of inflation [66, 67]. There, breaking of spatial diffeomorphism was considered by effective quadratic mass terms or derivative operators for metric fluctuations in the Lagrangian during inflation without the necessity for specifying the UV completion, while noting that it may be a version of massive gravity coupled to an inflaton, some model of inflation using vectors, or sets of scalars obeying some symmetries.

Before closing this section, let us emphasize one important assumption made throughout this paper. We calculate evolution of primordial fluctuations assuming they obey general relativity below some energy scale. That energy scale and comoving wave number $k$ of primordial fluctuations are related as follows. The wave number $k$ is said to reenter the horizon when $k=a H$, where $a$ and $H$ are the scale factor and the Hubble parameter. The scale factor can be eliminated by the relation $H^{2}=H_{0}^{2} \Omega_{r} a^{-4}$, where $\Omega_{r}$ is the radiation density parameter and $H_{0}$ is the current Hubble parameter and here they are taken as $\Omega_{r}=5 \times 10^{-5}$ and $H_{0}=67 \mathrm{~km} / \mathrm{s} / \mathrm{Mpc}$. The Hubble parameter $H$ and the temperature of the Universe $T$ are related by (in natural units) $H^{2}=4 \pi^{3} g_{*} T^{4} / 45$, where $g_{*}$ is the degrees of freedom of relativistic species here taken as $g_{*}=106.75$. From these relations the temperature and comoving wave number are related by

$$
T=\left(\frac{4 \pi^{3}}{45} g_{*}\right)^{-\frac{1}{4}}\left(H_{0} \Omega_{r}^{1 / 2} k^{-2}\right)^{-\frac{1}{2}} \simeq 5 \times 10^{10} \mathrm{GeV}\left(\frac{k}{10^{18} \mathrm{Mpc}^{-1}}\right) .
$$

For instance, if the theory is reduced to the standard cosmology described by general relativity at $T=5 \times 10^{10} \mathrm{GeV}$, then our upper limits summarized in Fig. 4 are applicable for $k<10^{18} \mathrm{Mpc}^{-1}$. 


\section{RADIATION DENSITY PERTURBATIONS GENERATED FROM TENSOR PERTURBATIONS}

We work in the comoving gauge, in which the metric is written as ${ }^{\dagger 4}$

$$
d s^{2}=a^{2}\left[-(1+2 \Phi) d \eta^{2}+2 B_{, i} d \eta d x^{i}+\left((1-2 \Psi) \delta_{i j}+2 h_{i j}\right) d x^{i} d x^{j}\right]
$$

where $h_{i j}$ is the tensor perturbation satisfying $h_{i j, i}=h_{i i}=0$. Throughout this paper it is assumed that the amplitude of initial tensor perturbations is much larger than that of scalar perturbations (schematically, (scalar) $\ll$ (tensor)), and so the scalar quantities in the metric above should be regarded as second order in $h_{i j}$. Hence, for scalar perturbations we write down the Einstein equations keeping second-order terms only in $h_{i j}$. As is also mentioned in the Introduction, our upper bounds from PBHs on tensor perturbations thus obtained are applicable even if this initial hierarchy between tensor and scalar perturbations does not hold. This is because if the amplitude of scalar perturbations is as larger as, or larger than that of tensor perturbations, then the abundance of PBHs increases when the amplitude of tensor modes is fixed. Namely, assuming (scalar) « (tensor) initially is most conservative in placing upper bounds on tensor modes, and hence our bounds are applicable even if that assumption does not hold.

Let us write down the fundamental equations in the following. We denote the energy density and pressure of the dominating radiation by $\rho$ and $p$, respectively, and write $p=c_{\mathrm{S}}^{2} \rho$, where $c_{\mathrm{s}}$ is the speed of sound. In this paper we restrict our attention to the formation of PBHs due to collapse of radiation density perturbations during the radiation-dominated era, and so we set $c_{\mathrm{s}}=1 / \sqrt{3}$ in calculations, though we leave $c_{\mathrm{s}}$ unspecified in equations below for generality. We decompose $\rho$ and $p$ as $\rho(\eta, \underline{\mathbf{x}})=\rho_{0}(\eta)+\delta \rho(\eta, \boldsymbol{x})$ and $p(\eta, \boldsymbol{x})=p_{0}(\eta)+\delta p(\eta, \boldsymbol{x})$.

The zeroth-order Einstein equations yield

$$
\begin{aligned}
\mathcal{H}^{2} & =\frac{8 \pi G}{3} a^{2} \rho_{0}, \\
\mathcal{H}^{2}-\mathcal{H}^{\prime} & =4 \pi G a^{2}\left(\rho_{0}+p_{0}\right),
\end{aligned}
$$

where $\mathcal{H} \equiv a^{\prime} / a$ with the prime denoting differentiation with respect to the conformal time $\eta$. These two equations are combined to give

$$
2 \mathcal{H}^{\prime}+\left(1+3 c_{\mathrm{s}}^{2}\right) \mathcal{H}^{2}=0 .
$$

$\dagger 4$ Perturbations to the metric and energy momentum tensor are written as (see [68] for more details)

$$
\begin{gathered}
d s^{2}=a^{2}\left[-(1+2 \Phi) d \eta^{2}+2 B_{, i} d \eta d x^{i}+\left\{(1-2 \Psi) \delta_{i j}-2 E_{, i j}-2 h_{i j}\right\} d x^{i} d x^{j}\right], \\
T_{\mu \nu}=(p+\delta p) g_{\mu \nu}+(\rho+\delta \rho+p+\delta p)\left(u_{\mu}+\delta u_{\mu}\right)\left(u_{\nu}+\delta u_{\nu}\right),
\end{gathered}
$$

where the spatial components of the velocity perturbation $\delta u_{\mu}$ are written as $\delta u_{i}=\delta u_{, i}$. Let us consider a coordinate transformation of the form $x^{\mu} \rightarrow x^{\mu}+\epsilon^{\mu}\left(x^{\mu}\right)$, with $\epsilon_{0}=-\epsilon^{0}, \epsilon_{i}=a^{2} \epsilon^{i}, \epsilon_{i}=\epsilon_{, i}$. Then $E$ and $\delta u$ transform as $E \rightarrow E+\epsilon / a^{2}, \delta u \rightarrow \delta u-\epsilon_{0}$. Here we choose $\epsilon$ so that $E=0$, and then choose $\epsilon_{0}$ so that $\delta u=0$. Both choices are unique, so that there is no freedom to make further gauge transformations. This choice is sometimes called the comoving gauge (e.g. [69]). 
The Einstein equations at first order in $h_{i j}$ give the standard evolution equation for tensor modes as follows:

$$
h_{i j}^{\prime \prime}+2 \mathcal{H} h_{i j}^{\prime}-\Delta h_{i j}=0 .
$$

The Einstein equations at second order in $h_{i j}$, derived in Appendix A, are as follows:

$$
\begin{gathered}
\Delta \Psi-3 \mathcal{H}\left(\Psi^{\prime}+\mathcal{H} \Phi\right)-\mathcal{H} \Delta B+S_{1}=4 \pi G a^{2} \delta \rho, \\
\left(\Psi^{\prime}+\mathcal{H} \Phi+S_{2}\right)_{, i}=0 \\
\Psi^{\prime \prime}+\mathcal{H}(2 \Psi+\Phi)^{\prime}+\left(2 \mathcal{H}^{\prime}+\mathcal{H}^{2}\right) \Phi+\frac{1}{2} \Delta\left(\Phi-\Psi+B^{\prime}+2 \mathcal{H} B\right)+S_{3}+S_{4}=4 \pi G a^{2} \delta p \\
\left(\Phi-\Psi+B^{\prime}+2 \mathcal{H} B-2 S_{5}\right)_{, i j}=0 .
\end{gathered}
$$

In these equations the following terms, second order in $h_{i j}$, source the scalar perturbations:

$$
\begin{gathered}
S_{1} \equiv-\frac{1}{4} h_{i j}^{\prime} h^{i j^{\prime}}-2 \mathcal{H} h_{i j} h^{i j^{\prime}}+h_{i j} \Delta h^{i j}-\frac{1}{2} \partial_{j} h_{i k} \partial^{k} h^{i j}+\frac{3}{4} \partial_{k} h_{i j} \partial^{k} h^{i j}, \\
\Delta S_{2}=\partial^{i} S_{i}, \quad S_{i}=-h^{j k} \partial_{k} h_{i j}^{\prime}+\frac{1}{2} h^{j k^{\prime}} \partial_{i} h_{j k}+h^{j k} \partial_{i} h_{j k}^{\prime}, \\
S_{3} \equiv \frac{3}{4} h_{i j}^{\prime} h^{i j^{\prime}}+h_{i j} h^{i j^{\prime \prime}}+2 \mathcal{H} h_{i j} h^{i j^{\prime}}-h_{i j} \Delta h^{i j}+\frac{1}{2} \partial_{j} h_{i k} \partial^{k} h^{i j}-\frac{3}{4} \partial_{k} h_{i j} \partial^{k} h^{i j}, \\
\Delta S_{4}=\frac{1}{2}\left(\Delta S^{i}{ }_{i}-\partial^{i} \partial^{j} S_{i j}\right), \\
\Delta^{2} S_{5}=\frac{1}{2}\left(3 \partial^{i} \partial^{j} S_{i j}-\Delta S_{i}^{i}\right), \\
S_{i j} \equiv-h_{i}{ }^{k^{\prime}} h_{j k}^{\prime}-h_{i k} h_{j}{ }^{k^{\prime \prime}}-2 \mathcal{H} h_{i}{ }^{k} h_{j k}^{\prime}+h^{k l} \partial_{k} \partial_{l} h_{i j}+h_{i}{ }^{k} \Delta h_{j k}-h^{k l} \partial_{l} \partial_{i} h_{j k}-h^{k l} \partial_{l} \partial_{j} h_{i k} \\
-\partial_{k} h_{j l} \partial^{l} h_{i}{ }^{k}+\partial_{l} h_{j k} \partial^{l} h_{i}{ }^{k}+\frac{1}{2} \partial_{i} h_{k l} \partial_{j} h^{k l}+h^{k l} \partial_{i} \partial_{j} h_{k l} .
\end{gathered}
$$

Using (3.7), $S_{1}, S_{3}$ and $S_{i j}$ are rewritten as follows:

$$
\begin{gathered}
S_{1}=-\frac{1}{4} h_{i j}^{\prime} h^{i j^{\prime}}+h_{i j} h^{i j^{\prime \prime}}-\frac{1}{2} \partial_{j} h_{i k} \partial^{k} h^{i j}+\frac{3}{4} \partial_{k} h_{i j} \partial^{k} h^{i j}, \\
S_{3}=\frac{3}{4} h_{i j}^{\prime} h^{i j^{\prime}}+\frac{1}{2} \partial_{j} h_{i k} \partial^{k} h^{i j}-\frac{3}{4} \partial_{k} h_{i j} \partial^{k} h^{i j} \\
S_{i j}=-h_{i}{ }^{k^{\prime}} h_{j k}^{\prime}+h^{k l} \partial_{k} \partial_{l} h_{i j}-h^{k l} \partial_{l} \partial_{i} h_{j k}-h^{k l} \partial_{l} \partial_{j} h_{i k} \\
-\partial_{k} h_{j l} \partial^{l} h_{i}{ }^{k}+\partial_{l} h_{j k} \partial^{l} h_{i}{ }^{k}+\frac{1}{2} \partial_{i} h_{k l} \partial_{j} h^{k l}+h^{k l} \partial_{i} \partial_{j} h_{k l} .
\end{gathered}
$$

The conservation of the energy-momentum tensor yields

$$
\begin{gathered}
\delta \rho^{\prime}+3 \mathcal{H}(\delta \rho+\delta p)-(\rho+p) \Delta B-3(\rho+p) \Psi^{\prime}-2(\rho+p) h^{i j} h_{i j}^{\prime}=0, \\
\partial_{i}(\delta p+(\rho+p) \Phi)=0 .
\end{gathered}
$$

From these equations one can derive the evolution equation of $\Psi$ as follows. First, Eqs. (3.21) and (3.22) lead to (hereafter we work in Fourier space)

$$
\Phi^{\prime}=-c_{s}^{2}\left(-k^{2} B+3 \Psi^{\prime}+2 h^{i j} h_{i j}^{\prime}\right) .
$$


The term $-k^{2} B$ of the above can be eliminated by the following relation, obtained from Eqs. (3.8) and (3.9):

$$
-k^{2} B=\frac{-k^{2} \Psi}{\mathcal{H}}+3 S_{2}+\frac{S_{1}}{\mathcal{H}}-\frac{3}{2} \mathcal{H} \delta_{r},
$$

where $\delta_{r} \equiv \delta \rho / \rho_{0}$. Using these and (3.11) as well as (3.6), (3.10) can be rewritten as

$$
\Psi^{\prime \prime}+2 \mathcal{H} \Psi^{\prime}+c_{\mathrm{S}}^{2} k^{2} \Psi=S .
$$

Here,

$$
S \equiv c_{\mathrm{s}}^{2} S_{1}-S_{3}-\hat{k}^{i} \hat{k}^{j} S_{i j}+2 c_{\mathrm{s}}^{2} \mathcal{H} h^{i j} h_{i j}^{\prime}
$$

is the source term representing generation of scalar perturbations due to the tensor perturbations. From (3.9) and (3.22), the energy density perturbation is given by

$$
\delta_{r}=\frac{1+c_{\mathrm{s}}^{2}}{c_{s}^{2} \mathcal{H}}\left(\Psi^{\prime}+S_{2}\right) .
$$

Eq. (3.25) can be formally solved as ${ }^{\dagger 5}$

$$
\Psi(\eta, \boldsymbol{k})=a^{-1}(\eta) \int_{0}^{\eta} d \tilde{\eta} g_{k}(\eta, \tilde{\eta}) a(\tilde{\eta}) S(\tilde{\eta}, \boldsymbol{k}),
$$

where $g_{k}$ is the retarded Green's function satisfying

$$
g_{k}^{\prime \prime}+\left(c_{\mathrm{s}}^{2} k^{2}-\frac{a^{\prime \prime}}{a}\right) g_{k}=\delta(\eta-\tilde{\eta}) .
$$

During the radiation-dominated epoch, its solution can be constructed by the two homogeneous solutions

$$
v_{1}(k, \eta)=\sin \left(c_{\mathrm{s}} k \eta\right), \quad v_{2}(k, \eta)=\cos \left(c_{\mathrm{s}} k \eta\right)
$$

as follows [45]:

$$
g_{k}(\eta, \tilde{\eta})=\frac{v_{1}(k, \eta) v_{2}(k, \tilde{\eta})-v_{1}(k, \tilde{\eta}) v_{2}(k, \eta)}{v_{1}^{\prime}(k, \tilde{\eta}) v_{2}(k, \tilde{\eta})-v_{1}(k, \tilde{\eta}) v_{2}^{\prime}(k, \tilde{\eta})}=\frac{1}{c_{\mathrm{s}} k} \sin \left(c_{\mathrm{s}} k(\eta-\tilde{\eta})\right) \text { for } \eta \geq \tilde{\eta} .
$$

The two point correlation function of $\Psi$ can be expressed as, denoting its nondimensional power spectrum by $\mathcal{P}_{\Psi}$,

$$
\begin{aligned}
& \left\langle\Psi(\eta, \boldsymbol{k}) \Psi^{*}(\eta, \boldsymbol{K})\right\rangle=\frac{2 \pi^{2}}{k^{3}} \delta(\boldsymbol{k}-\boldsymbol{K}) \mathcal{P}_{\Psi}(k) \\
& =a^{-2}(\eta) \int_{0}^{\eta} d \eta_{1} \int_{0}^{\eta} d \eta_{2} g_{k}\left(\eta, \eta_{1}\right) g_{K}\left(\eta, \eta_{2}\right) a\left(\eta_{1}\right) a\left(\eta_{2}\right)\left\langle S\left(\eta_{1}, \boldsymbol{k}\right) S\left(\eta_{2}, \boldsymbol{K}\right)\right\rangle .
\end{aligned}
$$

$\dagger 5$ We choose $\eta=0$ at the beginning of the radiation-dominated era, and we assume the initial condition is $\Psi(0, \boldsymbol{k})=0$. Strictly speaking, however, $\Psi$ is also generated before the radiation-dominated era at second order in tensor perturbations, even without intrinsic first-order scalar perturbations. That generation is highly model-dependent, and hence we restrict attention to the generation of $\Psi$ only during the radiationdominated era to adopt the above initial condition. This neglect of the generation of $\Psi$ before the radiation-dominated era would probably lead to conservative upper bounds on tensor perturbations, since in general $\Psi$ would be larger if the generation before $\eta=0$ is additionally taken into account. An analogous assumption is also made in the literature discussing induced gravitational waves (see footnote†2). 
In the following, let us write down the Fourier components of the source $S$, given by (3.26). We begin by decomposing $h_{i j}(\eta, \boldsymbol{x})$ as (following [49]):

$$
h_{i j}(\eta, \boldsymbol{x})=\int \frac{d^{3} \boldsymbol{k}}{(2 \pi)^{3 / 2}} e^{i \boldsymbol{k} \cdot \boldsymbol{x}}\left(h^{+}(\eta, \boldsymbol{k}) e_{i j}^{+}(\boldsymbol{k})+h^{\times}(\eta, \boldsymbol{k}) e_{i j}^{\times}(\boldsymbol{k})\right),
$$

where for $\boldsymbol{k}$ in the z-direction

$$
e_{11}^{+}(\hat{z})=-e_{22}^{+}(\hat{z})=e_{12}^{\times}(\hat{z})=e_{21}^{\times}(\hat{z})=1, \quad \text { others }=0
$$

while for $\hat{k} \equiv \boldsymbol{k} /|\boldsymbol{k}|$ in any other direction, $e_{i j}^{r}(\hat{k})(r=+, \times)$ is defined by applying on each of the indices $i$ and $j$ a standard rotation, that takes the z-direction into the direction of $\hat{k}$ (see e.g. [68]). Then one can check the following:

$$
\sum_{i j} e_{i j}^{r}(\boldsymbol{k}) e_{i j}^{s}(\boldsymbol{k})=2 \delta^{r s}
$$

Let us further decompose the Fourier components as $h^{r}(\eta, \boldsymbol{k})=D(\eta, k) h^{r}(\boldsymbol{k})$, where $h^{r}(\boldsymbol{k})$ is the initial amplitude and $D(\eta, k)$ is the growth factor, which can be obtained by solving the linear evolution equation (3.7) for $h_{i j}$ (dropping the decaying mode):

$$
D(\eta, k)=\frac{\sin k \eta}{k \eta}
$$

It turns out that the Fourier components of the source $S$ can be written as follows (see Appendix $\mathrm{B})$ :

$$
\begin{gathered}
S(\eta, \boldsymbol{k})=\sum_{r s} \int \frac{d^{3} \boldsymbol{k}^{\prime}}{(2 \pi)^{3 / 2}} h^{r}\left(\boldsymbol{k}^{\prime}\right) h^{s}\left(\boldsymbol{k}-\boldsymbol{k}^{\prime}\right) A_{r s}\left(\eta, \boldsymbol{k}, \boldsymbol{k}^{\prime}\right), \\
A_{r s}\left(\eta, \boldsymbol{k}, \boldsymbol{k}^{\prime}\right) \equiv f_{1}\left(\eta, \boldsymbol{k}, \boldsymbol{k}^{\prime}\right) E_{1}^{r s}\left(\boldsymbol{k}, \boldsymbol{k}^{\prime}\right)+f_{2}\left(\eta, \boldsymbol{k}, \boldsymbol{k}^{\prime}\right) E_{2}^{r s}\left(\boldsymbol{k}, \boldsymbol{k}^{\prime}\right) .
\end{gathered}
$$

Here,

$$
E_{1}^{r s}\left(\boldsymbol{k}, \boldsymbol{k}^{\prime}\right) \equiv \hat{k}_{j} \hat{k}^{k} e_{i k}^{r}\left(\boldsymbol{k}^{\prime}\right) e_{s}^{i j}\left(\boldsymbol{k}-\boldsymbol{k}^{\prime}\right), \quad E_{2}^{r s}\left(\boldsymbol{k}, \boldsymbol{k}^{\prime}\right) \equiv e_{r}^{i j}\left(\boldsymbol{k}^{\prime}\right) e_{i j}^{s}\left(\boldsymbol{k}-\boldsymbol{k}^{\prime}\right),
$$

and their nonzero components are written as ${ }^{\dagger 6}$

$$
\begin{aligned}
E_{1}^{++}\left(\boldsymbol{k}, \boldsymbol{k}^{\prime}\right)=- & \mu_{1} \sqrt{1-\mu^{2}} \sqrt{1-\mu_{2}^{2}}, \quad E_{1}^{\times \times}\left(\boldsymbol{k}, \boldsymbol{k}^{\prime}\right)=-\sqrt{1-\mu^{2}} \sqrt{1-\mu_{2}^{2}}, \\
E_{2}^{++}\left(\boldsymbol{k}, \boldsymbol{k}^{\prime}\right)=1+\mu_{1}^{2}, & E_{2}^{\times \times}\left(\boldsymbol{k}, \boldsymbol{k}^{\prime}\right)=2 \mu_{1},
\end{aligned}
$$

where $\mu \equiv \boldsymbol{k} \cdot \boldsymbol{k}^{\prime} / k k^{\prime}$ and

$$
\mu_{1} \equiv \frac{\boldsymbol{k}^{\prime} \cdot\left(\boldsymbol{k}-\boldsymbol{k}^{\prime}\right)}{k^{\prime}\left|\boldsymbol{k}-\boldsymbol{k}^{\prime}\right|}=\frac{k \mu-k^{\prime}}{\left|\boldsymbol{k}-\boldsymbol{k}^{\prime}\right|}, \quad \mu_{2} \equiv \frac{\boldsymbol{k} \cdot\left(\boldsymbol{k}-\boldsymbol{k}^{\prime}\right)}{k\left|\boldsymbol{k}-\boldsymbol{k}^{\prime}\right|}=\frac{k-k^{\prime} \mu}{\left|\boldsymbol{k}-\boldsymbol{k}^{\prime}\right|} .
$$

Also the above $f_{1}$ and $f_{2}$ are given by (see Appendix B)

$$
\begin{array}{r}
f_{1}\left(\eta, \boldsymbol{k}, \boldsymbol{k}^{\prime}\right)=D\left(\eta, k^{\prime}\right)\left\{\overleftarrow{\partial_{\eta}} \partial_{\eta}-\frac{1}{2}\left(3-c_{\mathrm{s}}^{2}\right) k^{2}+3 k k^{\prime} \mu-k^{\prime 2}\right\} D\left(\eta,\left|\boldsymbol{k}-\boldsymbol{k}^{\prime}\right|\right) \\
f_{2}\left(\eta, \boldsymbol{k}, \boldsymbol{k}^{\prime}\right)=D\left(\eta, k^{\prime}\right)\left\{-\frac{1}{4}\left(3+c_{\mathrm{s}}^{2}\right) \overleftarrow{\partial_{\eta}} \partial_{\eta}+c_{\mathrm{s}}^{2} \partial_{\eta}^{2}+2 c_{\mathrm{s}}^{2} \mathcal{H} \partial_{\eta}+\frac{1}{8}\left(1-3 c_{\mathrm{s}}^{2}\right) k^{2}\right. \\
\left.-\frac{1}{2} k^{\prime} \mu\left(k-k^{\prime} \mu\right)+\frac{3}{4}\left(1+c_{\mathrm{s}}^{2}\right) k^{\prime 2}\right\} D\left(\eta,\left|\boldsymbol{k}-\boldsymbol{k}^{\prime}\right|\right)
\end{array}
$$

†6 These expressions are obtained by first setting $\hat{k}=\hat{z}$, which is possible due to isotropy, and by assuming $\hat{k}^{\prime}$ is on the $z-y$ plane, which is justified by the rotational invariance of $E_{1}^{r s}$ and $E_{2}^{r s}$. 
where $\overleftarrow{\partial_{\eta}}$ is supposed to differentiate only $D\left(\eta, k^{\prime}\right)$ in the left.

Introducing the power spectrum of tensor perturbations as

$$
\left\langle h^{r}(\boldsymbol{k}) h^{s *}(\boldsymbol{K})\right\rangle=\frac{2 \pi^{2}}{k^{3}} \delta(\boldsymbol{k}-\boldsymbol{K}) \delta_{r s} \mathcal{P}_{h}(k)
$$

and assuming $h^{r}(\boldsymbol{k})$ is Gaussian, we can obtain the following expression for the correlation of the source:

$$
\left\langle S\left(\eta_{1}, \boldsymbol{k}\right) S\left(\eta_{2}, \boldsymbol{K}\right)\right\rangle=\pi \delta(\boldsymbol{k}+\boldsymbol{K}) \sum_{r s} \int d^{3} \boldsymbol{k}^{\prime} \frac{\mathcal{P}_{h}\left(k^{\prime}\right) \mathcal{P}_{h}\left(\left|\boldsymbol{k}-\boldsymbol{k}^{\prime}\right|\right)}{k^{\prime 3}\left|\boldsymbol{k}-\boldsymbol{k}^{\prime}\right|^{3}} A_{r s}\left(\eta_{1}, \boldsymbol{k}, \boldsymbol{k}^{\prime}\right) A_{r s}\left(\eta_{2}, \boldsymbol{k}, \boldsymbol{k}^{\prime}\right) .
$$

In this paper, we assume the following delta-function-type tensor power spectrum:

$$
\mathcal{P}_{h}(k)=\mathcal{A}^{2} k \delta\left(k-k_{p}\right) .
$$

From (3.27) and (3.28), the energy density perturbation can be calculated as

$$
\begin{aligned}
\delta_{r}(\eta, \boldsymbol{k})=\frac{1+c_{\mathrm{s}}^{2}}{c_{\mathrm{s}}^{2} \mathcal{H}} \sum_{r s} \int \frac{d^{3} \boldsymbol{k}^{\prime}}{(2 \pi)^{3 / 2}} h^{r}\left(\boldsymbol{k}^{\prime}\right) h^{s}\left(\boldsymbol{k}-\boldsymbol{k}^{\prime}\right) F_{r s}\left(\eta, \boldsymbol{k}, \boldsymbol{k}^{\prime}\right) \\
F_{r s}\left(\eta, \boldsymbol{k}, \boldsymbol{k}^{\prime}\right) \equiv \int d \tilde{\eta}(\tilde{\eta} / \eta) A_{r s}\left(\tilde{\eta}, \boldsymbol{k}, \boldsymbol{k}^{\prime}\right)\left(\partial_{\eta}-\mathcal{H}\right) g_{k}(\eta, \tilde{\eta}) \\
+D\left(\eta, k^{\prime}\right)\left\{-\partial_{\eta} E_{1}^{r s}+\left(\frac{1}{2} \overleftarrow{\partial_{\eta}}+\partial_{\eta}\right)\left(1-\frac{k^{\prime}}{k} \mu\right) E_{2}^{r s}\right\} D\left(\eta,\left|\boldsymbol{k}-\boldsymbol{k}^{\prime}\right|\right)
\end{aligned}
$$

The power spectrum is defined by

$$
\left\langle\delta_{r}(\eta, \boldsymbol{k}) \delta_{r}^{*}(\eta, \boldsymbol{K})\right\rangle_{\mathrm{C}} \equiv\left\langle\delta_{r}(\eta, \boldsymbol{k}) \delta_{r}^{*}(\eta, \boldsymbol{K})\right\rangle-\left\langle\delta_{r}(\eta, \boldsymbol{k})\right\rangle\left\langle\delta_{r}^{*}(\eta, \boldsymbol{K})\right\rangle=\frac{2 \pi^{2}}{k^{3}} \delta(\boldsymbol{k}-\boldsymbol{K}) \mathcal{P}_{\delta_{r}}(\eta, k)
$$

and is obtained as follows:

$$
\mathcal{P}_{\delta_{r}}(\eta, k)=\left(\frac{1+c_{\mathrm{s}}^{2}}{c_{\mathrm{s}}^{2}}\right)^{2} \mathcal{A}^{4}\left(\frac{k}{k_{p}}\right)^{2} \eta^{2} \Theta\left(1-\frac{k}{2 k_{p}}\right) \sum_{r s} F_{r s}\left(\eta, k, k_{p}, \frac{k}{2 k_{p}}\right)^{2} .
$$

The time evolutions of this power spectrum for a few modes are shown in Fig. 1, where $\mathcal{A}$ is set to unity. The power spectrum takes the maximum value shortly after the horizon crossing of each $k$ mode $(k \eta=1)$. After reaching the maximum, it starts oscillations with the amplitude almost constant, similarly to the behavior in the standard linear cosmological perturbation theory. This is because the tensor perturbations decay after the horizon crossing, and so do the source terms, and then our fundamental equations for scalar perturbations are reduced to the standard ones in the linear theory. 


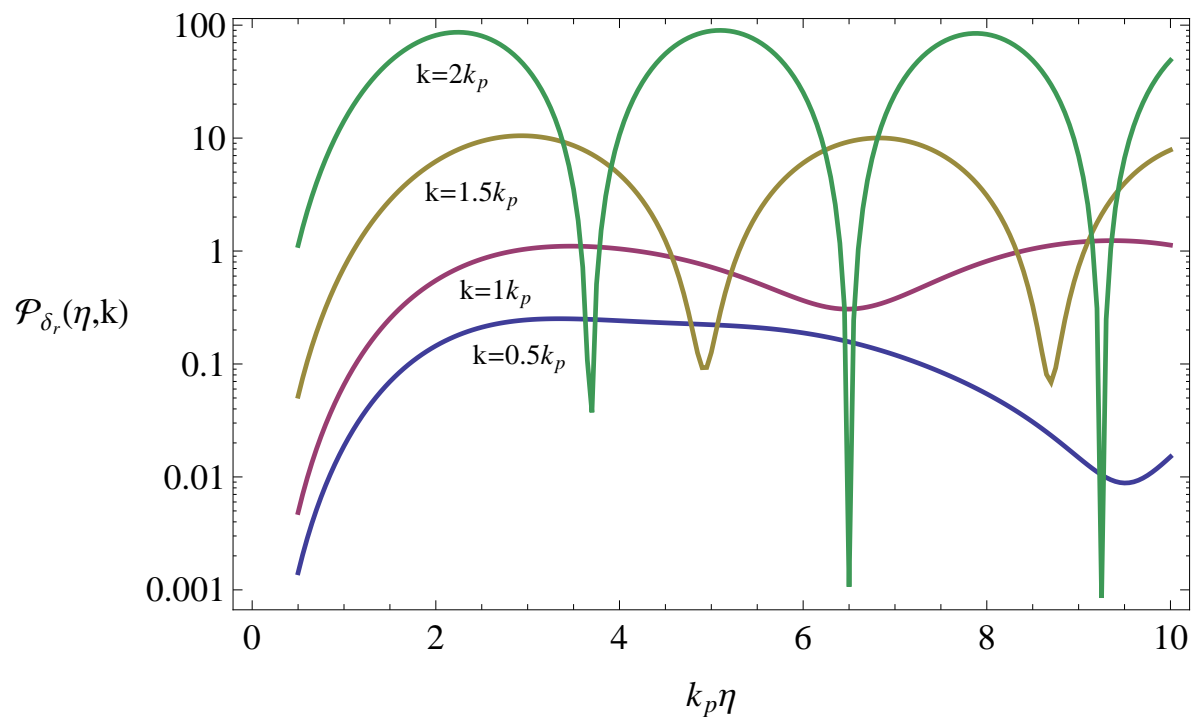

FIG. 1: The time evolution of the power spectrum for several modes, with $\mathcal{A}$ set to unity.

\section{UPPER BOUNDS ON PGWS FROM PBHS}

In order to place upper bounds on tensor modes from PBHs, the abundance of PBHs needs to be related to the primordial tensor power spectrum, which can be accomplished by integrating the probability density function (PDF) of the induced density perturbation averaged over the horizon. In the following we first estimate the moment when the PBH formation is most efficient for each $k_{p}$ by calculating the dispersion of the induced density perturbation, and then derive the PDF at this moment.

Let us begin by noting that the average $\left\langle\delta_{r}(\eta, \boldsymbol{x})\right\rangle$ is nonzero, since the density perturbation is generated by the tensor perturbations. To evaluate this average we introduce $f_{3}$ and $f_{4}$ by rewriting $F_{r s}$ as

$$
F_{r s}\left(\eta, \boldsymbol{k}, \boldsymbol{k}^{\prime}\right)=f_{3}\left(\eta, \boldsymbol{k}, \boldsymbol{k}^{\prime}\right) E_{1}^{r s}+f_{4}\left(\eta, \boldsymbol{k}, \boldsymbol{k}^{\prime}\right) E_{2}^{r s},
$$

where the explicit forms of $f_{3}$ and $f_{4}$ can be obtained by using (3.38), though the integration over $\eta$ can not be done analytically for general $k$ :

$$
\begin{gathered}
f_{3}\left(\eta, \boldsymbol{k}, \boldsymbol{k}^{\prime}\right)=\int d \tilde{\eta}(\tilde{\eta} / \eta) f_{1}\left(\tilde{\eta}, \boldsymbol{k}, \boldsymbol{k}^{\prime}\right)\left(\partial_{\eta}-\mathcal{H}\right) g_{k}(\eta, \tilde{\eta})-D\left(\eta, k^{\prime}\right) \partial_{\eta} D\left(\eta,\left|\boldsymbol{k}-\boldsymbol{k}^{\prime}\right|\right) \\
f_{4}\left(\eta, \boldsymbol{k}, \boldsymbol{k}^{\prime}\right)=\int d \tilde{\eta}(\tilde{\eta} / \eta) f_{2}\left(\tilde{\eta}, \boldsymbol{k}, \boldsymbol{k}^{\prime}\right)\left(\partial_{\eta}-\mathcal{H}\right) g_{k}(\eta, \tilde{\eta})+D\left(\eta, k^{\prime}\right)\left(\frac{1}{2} \overleftarrow{\partial_{\eta}}+\partial_{\eta}\right)\left(1-\frac{k^{\prime}}{k} \mu\right) D\left(\eta,\left|\boldsymbol{k}-\boldsymbol{k}^{\prime}\right|\right)
\end{gathered}
$$

Since only the zero-mode $\delta_{r}(\eta, \boldsymbol{k}=\mathbf{0})$ contributes to $\left\langle\delta_{r}(\eta, \boldsymbol{x})\right\rangle$, we need $f_{3}$ and $f_{4}$ only in the limit of $\boldsymbol{k} \rightarrow \mathbf{0}$, which are, under the assumption of the delta-function-type power spectrum (3.47),

$$
f_{3}=0, \quad f_{4}=-\frac{-1+2 k_{p}^{2} \eta^{2}+\cos \left(2 k_{p} \eta\right)}{24 k_{p}^{2} \eta^{3}} .
$$




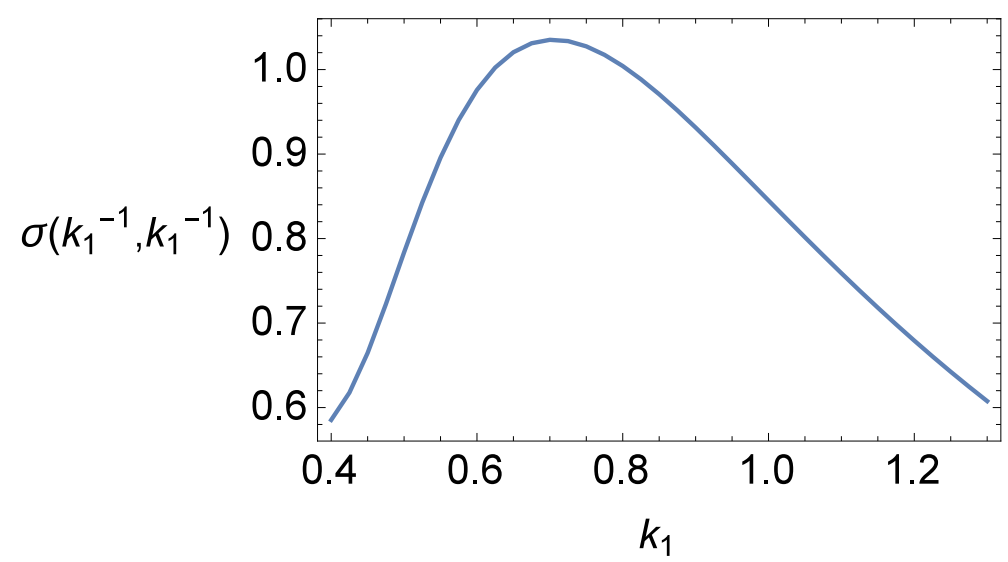

FIG. 2: The dependence of $\sigma\left(\eta=k_{1}^{-1}, R=k_{1}^{-1}\right)$ on $k_{1}$, with $\mathcal{A}=1$ and $k_{p}=1$.

Hence,

$$
\begin{aligned}
& \left\langle\delta_{r}(\eta, \boldsymbol{x}=\mathbf{0})\right\rangle \\
& =\int \frac{d \boldsymbol{k}^{3}}{(2 \pi)^{3 / 2}} \frac{1+c_{\mathrm{s}}^{2}}{c_{\mathrm{s}}^{2} \mathcal{H}} \int \frac{d^{3} \boldsymbol{k}^{\prime}}{(2 \pi)^{3 / 2}} \frac{2 \pi^{2}}{k_{p}^{3}} \delta(\boldsymbol{k}) \mathcal{A}^{2} k_{p} \delta\left(k^{\prime}-k_{p}\right) f_{4}\left(\eta, \boldsymbol{k}=\mathbf{0}, \boldsymbol{k}^{\prime}\right) \times(2-(-2)) \\
& =-\frac{\left(1+c_{\mathrm{s}}^{2}\right) \mathcal{A}^{2}}{6 c_{\mathrm{s}}^{2} k_{p}^{2} \eta^{2}}\left\{-1+2 k_{p}^{2} \eta^{2}+\cos \left(2 k_{p} \eta\right)\right\} .
\end{aligned}
$$

When $k_{p} \eta \gg 1$, the time average of this quantity asymptotes to

$$
\left\langle\delta_{r}\right\rangle=-\frac{\left(1+c_{\mathrm{s}}^{2}\right)}{3 c_{\mathrm{s}}^{2}} \mathcal{A}^{2},
$$

while $\left\langle\delta_{r}\right\rangle \rightarrow 0$ for $k_{p} \eta \rightarrow 0^{\dagger 7}$. We denote the density perturbation averaged over a sphere with comoving radius $R$ by $\delta_{r}(\eta, \boldsymbol{x}, R)$, the dispersion of which is related to the power spectrum as follows:

$$
\sigma(\eta, R) \equiv\left(\left\langle\delta_{r}(\eta, \boldsymbol{x}, R)^{2}\right\rangle-\left\langle\delta_{r}(\eta, \boldsymbol{x})\right\rangle^{2}\right)^{1 / 2}=\left(\int \frac{d k}{k} W^{2}(k R) \mathcal{P}_{\delta_{r}}(\eta, k)\right)^{1 / 2},
$$

where $W$ is the Fourier transform of the top-hat window function: $W(x)=3(\sin x-x \cos x) / x^{3}$. Figure 2 shows that the dispersion of the density perturbation at the horizon crossing of some mode $k_{1}$ smoothed over the horizon scale at that moment (namely, $\left.\eta=k_{1}^{-1}\right), \sigma\left(\eta=k_{1}^{-1}, R=k_{1}^{-1}\right.$ ), is maximum and is $\sim \mathcal{A}^{2}$ at around $k_{1} \sim 0.7 k_{p}$. That is, PBHs are formed most efficiently at around this moment, and therefore we restrict our attention to this moment in the following.

To determine the abundance of PBHs, the PDF of the density perturbations is necessary. Often the PDF of the density perturbations is assumed to be Gaussian, but in our problem it is highly non-Gaussian, since density perturbations are generated by tensor perturbations, whose statistical properties are assumed to be Gaussian. We can in principle determine the PDF of $\delta_{r}$ by randomly

\footnotetext{
${ }^{\dagger 7}$ Strictly speaking this effect may be taken into account in the background Friedmann equations (3.4) and (3.5), but $\mathcal{A}^{2}$ is mostly less than 0.1 from Fig. 4 , so the correction to the upper bounds would be $\sim 0.1$ at most, while a rigorous treatment of this effect would greatly complicate analysis. Hence we neglect this effect.
} 
generating the Fourier modes of GWs $\left\{h^{r}(\boldsymbol{k})\right\}$ repeatedly (for the details see Appendix C), whose result is shown in Fig. 3. The $\mathrm{PDF} P\left(\delta_{r}\right)$ of $\delta_{r}$ thus obtained turns out to be well approximated

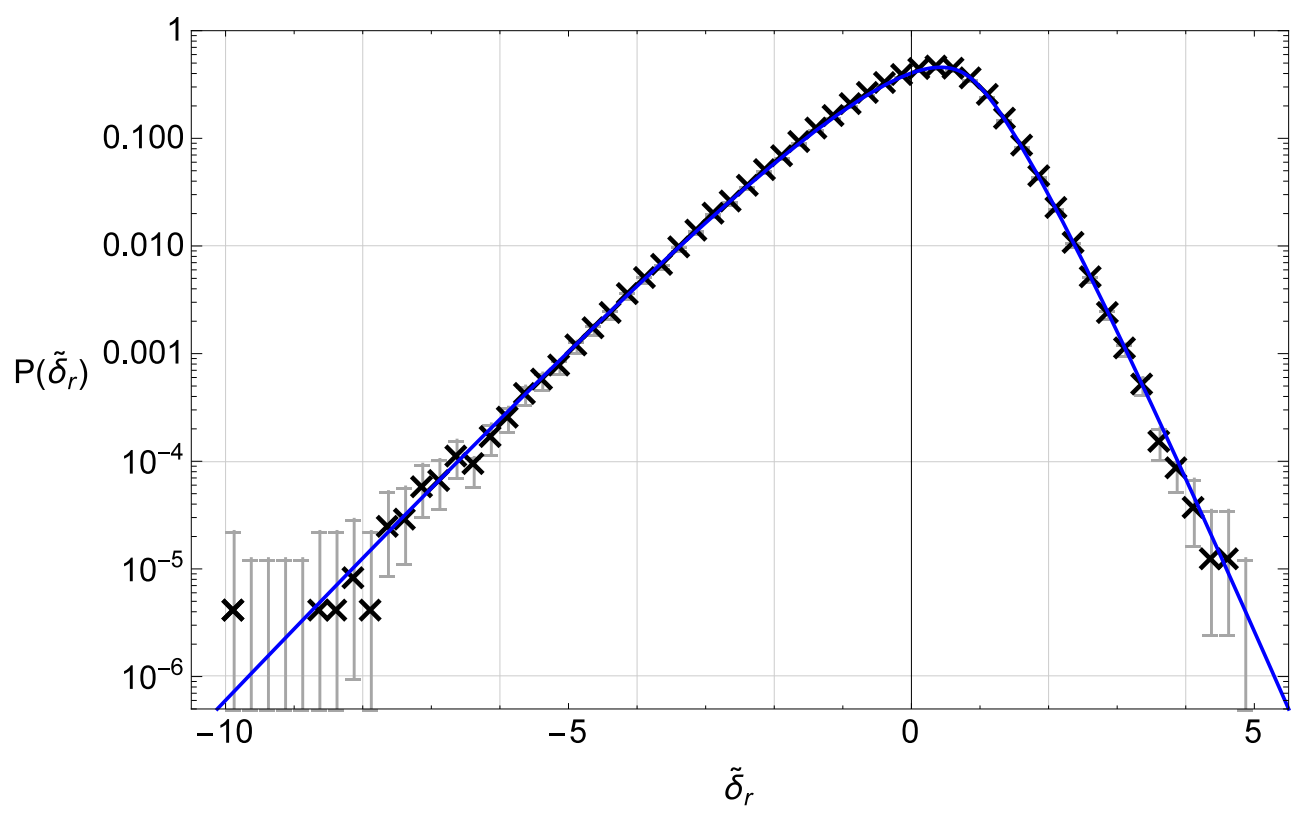

FIG. 3: The PDF of $\tilde{\delta}_{r} \equiv\left(\delta_{r}-\left\langle\delta_{r}\right\rangle\right) / \mathcal{A}^{2}$ for a million realizations of $\left\{h^{r}\left(\boldsymbol{k}_{i}\right)\right\}$ (see Appendix C for the details). The curve is the approximate PDF of $\tilde{\delta}_{r}$ given by (5.75).

by the formula (5.75). Then the fraction of the volume which has collapsed into PBHs at their formation is

$$
\beta=\int_{\delta_{r, \mathrm{th}}}^{\infty} \tilde{P}\left(\delta_{r}-\left\langle\delta_{r}\right\rangle\right) d \delta_{r}=\int_{\delta_{r, \mathrm{th}} / \mathcal{A}^{2}}^{\infty} P\left(\tilde{\delta}_{r}\right) d \tilde{\delta}_{r},
$$

where $\delta_{r, \text { th }}$ is the threshold of PBH formation, in the following assumed to be $\delta_{r, \text { th }}=0.4[20,22]^{\dagger 8}$. This quantity $\beta$ has been constrained on various masses and we use Fig. 9 of [24]. Then upper bounds on $\mathcal{A}^{2}$ for each $\beta$, corresponding to different masses of PBHs, are shown in Fig. 4, in which upper bounds are shown as a function of $k_{p}$, using the following relation between the PBH mass and the comoving wave number of perturbations:

$$
M_{\mathrm{PBH}}=2.2 \times 10^{13} M_{\odot}\left(\frac{k}{1 \mathrm{Mpc}^{-1}}\right)^{-2} .
$$

The dependence of the upper bounds on the comoving wave number is logarithmically weak owing to the exponential dependence of the PDF on $\delta_{r}$ and hence on $\mathcal{A}^{2}$ for $\delta_{r} \simeq \delta_{r \text {,th }}$.

$\dagger 8$ In these papers the initial conditions of numerical simulations were given in terms of curvature profiles in the limit of the vanishing ratio of the Hubble radius to the radius of perturbed regions. In the present work scalar perturbations are sourced by tensor perturbations, and hence strictly speaking the formation conditions obtained there may not be directly applied. A more precise treatment would require dedicated numerical simulations, which is beyond the scope of this work. The energy density of PGWs is expected to promote gravitational collapse, in light of previous works on direct collapse of nonlinear gravitational waves, mentioned in the footnote $\$ 3$ of the Introduction. This effect is not taken into account in the present paper, and therefore in this sense our upper bounds would be conservative. 


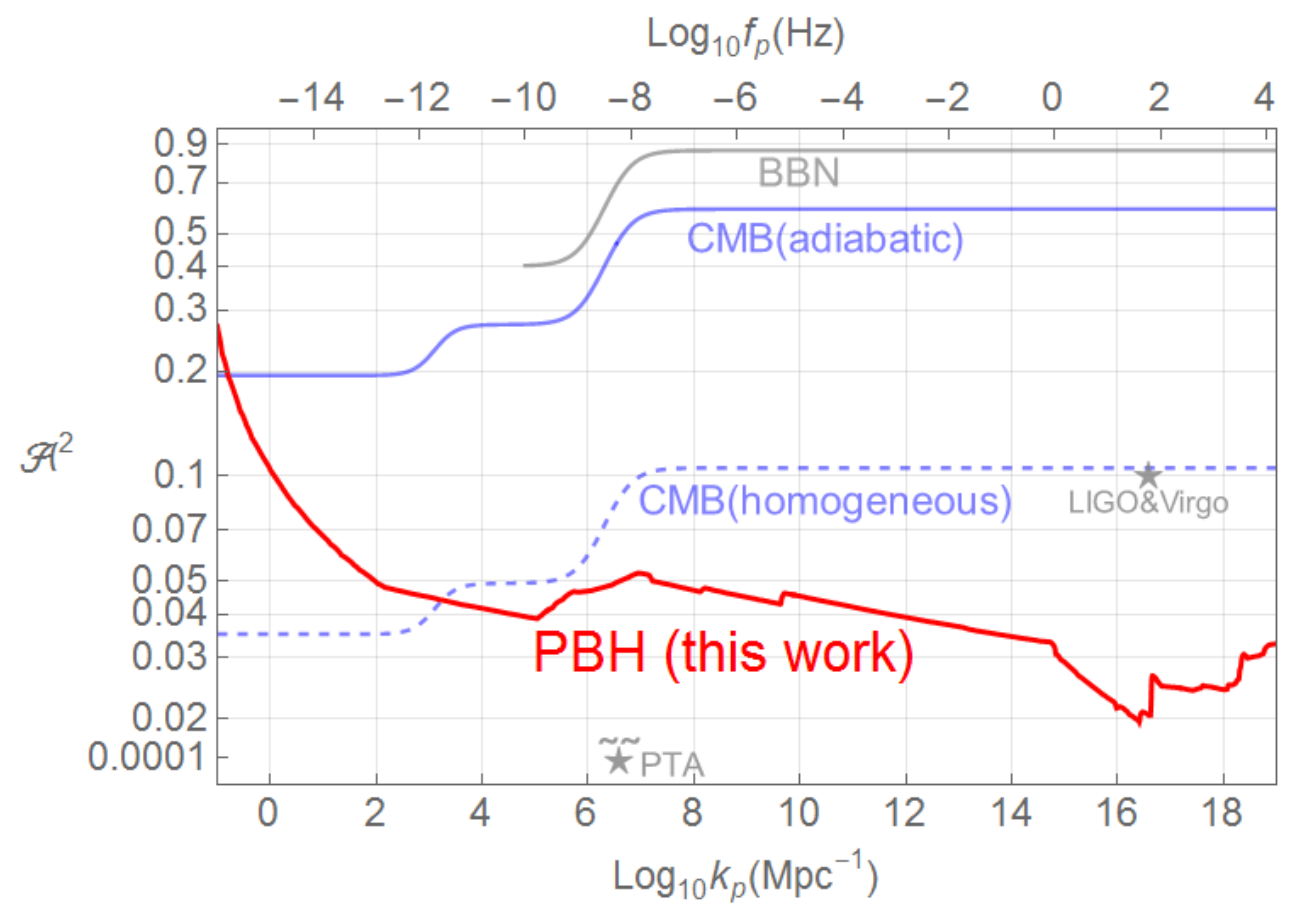

FIG. 4: Upper bounds on $\mathcal{A}^{2}$ as a function of $k_{p}$ using PBHs and other methods, also shown in [12].

Let us compare these PBH bounds with other bounds. We begin by rederiving the formula for the energy density of gravitational waves $\rho_{\mathrm{GW}}$ on subhorizon scales. Noting that $\rho_{\mathrm{GW}}=$ $-\left\langle S_{1}\right\rangle / 4 \pi G a^{2}$ from (3.8), where the brackets here imply temporal and spatial average (see e.g. [70] for more details), let us rewrite $\left\langle S_{1} / a^{2}\right\rangle$ in the following. By integration by parts and using (3.7),

$$
\begin{aligned}
\left\langle\frac{S_{1}}{a^{2}}\right\rangle & =\left\langle\frac{1}{a^{2}}\left(-\frac{1}{4} h_{i j}^{\prime} h^{i j^{\prime}}-\frac{3}{2} \mathcal{H} h_{i j} h^{i j^{\prime}}+\frac{1}{4} h_{i j} h^{i j^{\prime \prime}}\right)\right\rangle \\
& =\left\langle-\frac{1}{4} \dot{h}_{i j} \dot{h}^{i j}-\frac{5 \dot{a}}{4 a} h_{i j} \dot{h}^{i j}+\frac{1}{4} h_{i j} \ddot{h}^{i j}\right\rangle \simeq-\frac{1}{2}\left\langle\dot{h}_{i j} \dot{h}^{i j}\right\rangle,
\end{aligned}
$$

hence $^{\dagger 9}$,

$$
\rho_{\mathrm{GW}}=\frac{\left\langle\dot{h}_{i j} \dot{h}^{i j}\right\rangle}{8 \pi G} .
$$

Assuming the delta-function-type power spectrum (3.47),

$$
\rho_{\mathrm{GW}}=\frac{\mathcal{A}^{2}\left\langle\dot{D}\left(\eta, k_{p}\right)^{2}\right\rangle}{2 \pi G}=\frac{\mathcal{A}^{2}}{2 \pi G a^{2}}\left\langle\left(\frac{\cos k \eta}{\eta}-\frac{\sin k \eta}{k \eta^{2}}\right)^{2}\right\rangle \sim \frac{\mathcal{A}^{2}}{4 \pi G a^{2} \eta^{2}} .
$$

Defining $\rho_{\text {crit }} \equiv \rho_{\text {rad }}+\rho_{\mathrm{GW}} \simeq 3 H^{2} / 8 \pi G$, the following relation is obtained, used shortly:

$$
\frac{\rho_{\mathrm{GW}}}{\rho_{\mathrm{crit}}}=\frac{2}{3} \mathcal{A}^{2}
$$

${ }^{\dagger 9}$ If we define tensor perturbations without the factor 2 in front of $h_{i j}$ in (3.3), then we arrive at, instead of (4.11), the formula often used in the literature: $\rho_{\mathrm{GW}}=\left\langle\dot{h}_{i j} \dot{h}^{i j}\right\rangle / 32 \pi G$. 
The existence of gravitational waves is often effectively represented by the number of relativistic fermions' degrees of freedom as follows. First, the total energy density of radiation without gravitational waves nor dark radiation is written as

$$
\rho_{\mathrm{rad}}(T)=\frac{\pi^{2}}{30} g_{*} T^{4}
$$

where $g_{*}$ is the effective number of degrees of freedom of relativistic species and at the epoch of $\mathrm{BBN}$ it is given by $[3,71]$

$$
g_{*}=2+\frac{7}{8}\left\{4+2 N_{\nu}\right\}
$$

where $N_{\nu}$ is the effective number of degrees of freedom of neutrinos, $N_{\nu}=3.046^{\dagger 10}$. This is obtained by counting the degrees of freedom of photons, electrons, positrons, and neutrinos. At the photon decoupling, electrons and positrons should not be included. The presence of PGWs (or possibly of dark radiation) is represented by $\Delta N_{\text {eff }}$, as a correction to $N_{\nu}$ above. In the following we use $\Delta N_{\mathrm{GW}}$ as the contribution of PGWs and relate it to the primordial tensor power spectrum. When PGWs are present, the total energy density becomes (noting (4.6))

$$
\rho_{\text {tot }}=\rho_{\text {rad }}(T)\left(1+\left\langle\delta_{r}\right\rangle\right)+\rho_{\mathrm{GW}},
$$

which can be written, with the redefinition of the temperature $T \rightarrow T\left(1+\left\langle\delta_{r}\right\rangle / 4\right)$, as

$$
\rho_{\mathrm{tot}}=\rho_{\mathrm{rad}}(T)+\rho_{\mathrm{GW}} .
$$

After the horizon crossing of PGWs, $\rho_{\mathrm{GW}} \propto a^{-4}$, while, denoting by $g_{S}(T)$ the effective degrees of freedom of relativistic species in terms of entropy at temperature $T$, the photon temperature evolves according to $g_{S}(T) T^{3} a^{3}=$ const. (i.e. constant entropy) and therefore $\rho_{\text {rad }} \propto g_{*} T^{4} \sim 1 / a^{4} g_{S}^{1 / 3}$ (see e.g. [71]). Then, defining $\Omega_{\mathrm{GW}} \equiv \rho_{\mathrm{GW}} / \rho_{\text {crit }} \simeq \rho_{\mathrm{GW}} / \rho_{\mathrm{rad}}$,

$$
\Omega_{\mathrm{GW}}(T)=\left(\frac{g_{S}(T)}{g_{S}\left(T_{\mathrm{in}}\right)}\right)^{1 / 3} \Omega_{\mathrm{GW}}\left(T_{\mathrm{in}}\right)
$$

where $T_{\mathrm{in}}=T_{\mathrm{in}}\left(k_{p}\right)$ is the temperature of radiation when PGWs with comoving wave number $k_{p}$ reenter the horizon, and $T<T_{\mathrm{in}}^{\dagger 11}$. At the epoch of BBN, the contribution of PGWs is represented by $\Delta N_{\mathrm{GW}}$ as follows;

$$
\rho_{\mathrm{rad}}(T)+\rho_{\mathrm{GW}}(T)=\frac{\pi^{2}}{30}\left[2+\frac{7}{8}\left\{4+2\left(N_{\mathrm{sta}}+\Delta N_{\mathrm{GW}}\right)\right\}\right] T^{4},
$$

\footnotetext{
$\dagger 10$ The slight deviation from $N_{\nu}=3$ arises from the slight heating of neutrinos due to the relic interactions between $e^{ \pm}$and neutrinos at the epoch of $e^{ \pm}$annihilations, which took place only shortly after the neutrino decoupling [8].

$\dagger 11$ In [72] the following convenient fitting function is shown:
}

$$
g_{S}\left(T_{\mathrm{in}}(k)\right)=g_{S 0}\left\{\frac{A+\tanh \left[-2.5 \log _{10} k / 2 \pi f_{1}\right]}{A+1}\right\}\left\{\frac{B+\tanh \left[-2.0 \log _{10} k / 2 \pi f_{2}\right]}{B+1}\right\},
$$

where $A=\left(-1-g_{\mathrm{BBN}} / g_{S 0}\right) /\left(-1+g_{\mathrm{BBN}} / g_{S 0}\right), B=\left(-1-g_{\max } / g_{\mathrm{BBN}}\right) /\left(-1+g_{\max } / g_{\mathrm{BBN}}\right), g_{S 0}=3.91$, $g_{\mathrm{BBN}}=10.75, f_{1}=2.5 \times 10^{-12} \mathrm{~Hz}$ and $f_{2}=6.0 \times 10^{-9} \mathrm{~Hz}$. As for $g_{\max }$ following [72] we assume the sum of the Standard Model particles, $g_{\max }=106.75$. Note that $k / 2 \pi f_{1}=k /\left(1.6 \times 10^{-3} \mathrm{pc}^{-1}\right)$ and $k / 2 \pi f_{2}=k /\left(3.9 \mathrm{pc}^{-1}\right)$. 
which leads to

$$
\rho_{\mathrm{GW}}(T)=\rho_{\mathrm{rad}}(T) \times \frac{7}{8} \times 2 \times \Delta N_{\mathrm{GW}}(T) /\left\{2+\frac{7}{8}\left(4+2 N_{\mathrm{sta}}\right)\right\} \fallingdotseq \frac{7}{43} \rho_{\mathrm{rad}} \Delta N_{\mathrm{GW}}(T) .
$$

Since

$$
\Omega_{\mathrm{GW}}\left(T_{\mathrm{in}}\right) \simeq \frac{2}{3} \mathcal{A}^{2}
$$

from (4.13), $\Delta N_{\mathrm{GW}}(T)$ can be written as

$$
\Delta N_{\mathrm{GW}}(T)=\frac{43}{7} \Omega_{\mathrm{GW}}(T)=\frac{86}{21} \mathcal{A}^{2}\left(\frac{g_{S}(T)}{g_{S}\left(T_{\mathrm{in}}\right)}\right)^{1 / 3} .
$$

An upper bound on $\Delta N_{\text {eff }}, \Delta N_{\text {eff }}<\Delta N_{\text {upper }}$, is usually translated into an upper bound on $\Delta N_{\mathrm{GW}}$, $\Delta N_{\mathrm{GW}}<\Delta N_{\text {upper. }}$. As is also mentioned in the Introduction, in doing so we assume that any physical mechanisms, both known and unknown, increase $N_{\text {eff }}$, but at least there are examples where $N_{\text {eff }}$ decreases [9 11]. With this in mind, the requirement $\Delta N_{\mathrm{GW}}<\Delta N_{\text {upper }}$ is translated into an upper bound on $\mathcal{A}^{2}$ from (4.23) as follows:

$$
\mathcal{A}^{2} \lesssim \frac{21}{86}\left(\frac{g_{S}\left(T_{\text {in }}\right)}{g_{S}(T)}\right)^{1 / 3} \Delta N_{\text {upper }},
$$

with $g_{S}(T)=g_{\mathrm{BBN}}=10.75$.

On the other hand, at the photon decoupling,

$$
\rho_{\mathrm{rad}}(T)+\rho_{\mathrm{GW}}(T)=\frac{\pi^{2}}{30}\left\{2+2 \times \frac{7}{8}\left(\frac{4}{11}\right)^{4 / 3}\left(N_{\nu}+\Delta N_{\mathrm{GW}}\right)\right\},
$$

which yields

$$
\Omega_{\mathrm{GW}}(T)=\frac{2 \times \frac{7}{8}\left(\frac{4}{11}\right)^{4 / 3}}{2+2 \times \frac{7}{8}\left(\frac{4}{11}\right)^{4 / 3} N_{\nu}} \Delta N_{\mathrm{GW}} \simeq 0.13 \Delta N_{\mathrm{GW}} .
$$

So in this case we find

$$
\mathcal{A}^{2}<0.13 \times \frac{3}{2} \times \Delta N_{\text {upper }}\left(\frac{g_{S}\left(T_{\text {in }}\right)}{g_{S}(T)}\right)^{1 / 3},
$$

with $g_{S}(T)=g_{S 0}=3.91$. These constraints depend on $g_{S}\left(T_{\text {in }}\right)$, which one may regard as a drawback of these methods since it is uncertain especially at high temperatures. It is also potentially affected by some entropy production mechanisms [72]. On the other hand, the PBH constrains do not depend on $g_{S}$ nor other entropy productions much.

In order not to spoil the successful standard BBN, we follow [72] and set $\Delta N_{\text {upper }}=1.65$ as a $95 \%$ C.L. upper limit, which is applicable for the scales smaller than the comoving horizon atBBN, namely, $6.5 \times 10^{4} \mathrm{Mpc}^{-1} \lesssim k$.

As for CMB constraints, in [4] the use of homogeneous initial conditions of PGWs' energy density is advocated for those generated, for instance, by quantum fluctuations during inflation. In this case we use the $95 \%$ upper limit of $\Delta N_{\text {upper }}=0.18$ from $[73]^{\dagger 12}$. For adiabatic initial conditions of PGWs we refer to

$$
N_{\text {eff }}=3.52_{-0.45}^{+0.48} \quad\left(95 \% ; \text { Planck }+\mathrm{WP}+\text { highL }+\mathrm{H}_{0}+\mathrm{BAO}\right)
$$

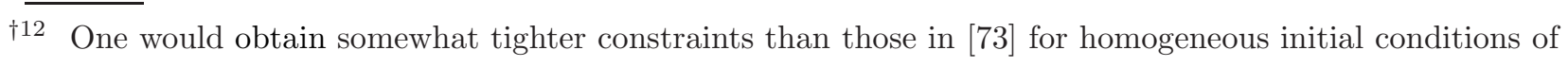
PGW' energy density, by repeating the analysis of [73] using more recent data. 


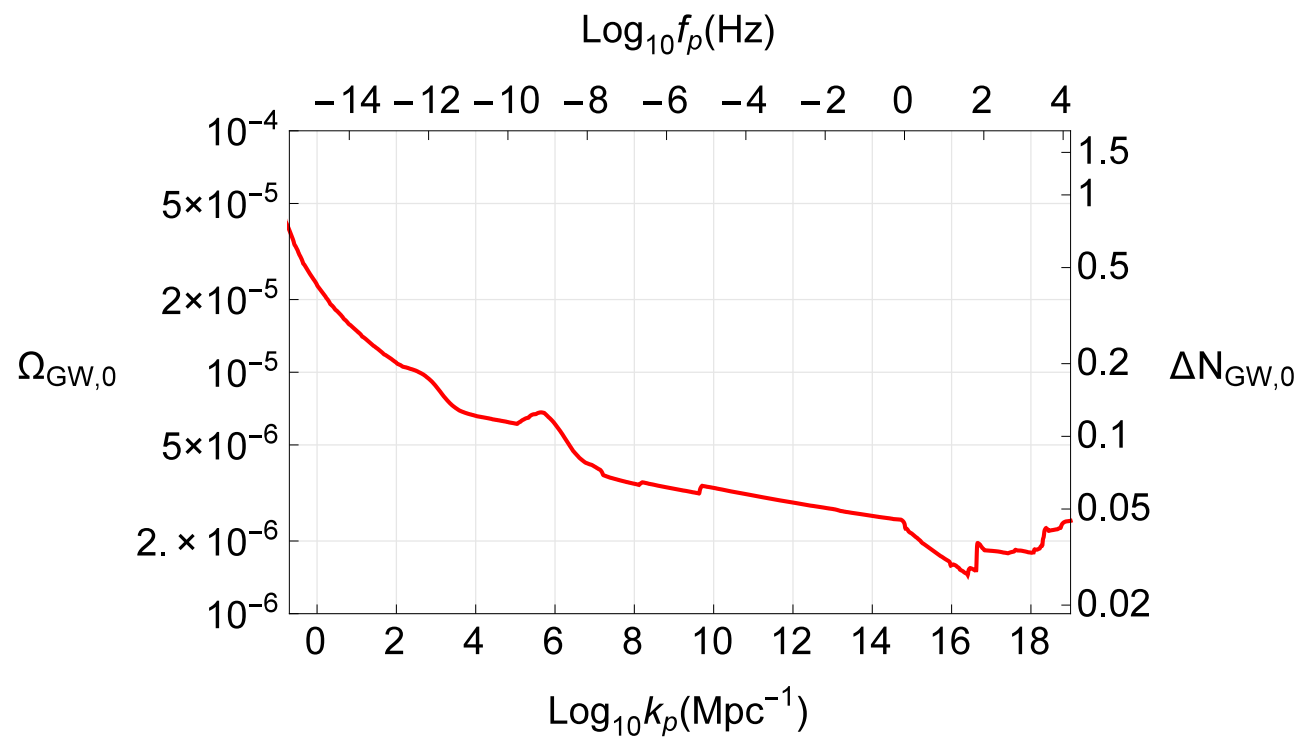

FIG. 5: Upper bounds from PBHs on $\Omega_{\mathrm{GW}, 0}$ or equivalently $\Delta N_{\mathrm{GW}, 0}$ as a function of $k_{p}$.

of [1] to set $\Delta N_{\text {upper }}=1.00$ [5].

The current energy density of PGWs, $\Omega_{\mathrm{GW}, 0}$, is also constrained by LIGO and Virgo, most severely in the band $41.5-169.25 \mathrm{~Hz}$ as $\Omega_{\mathrm{GW}, 0} \lesssim 5.6 \times 10^{-6} \times \log (169.25 / 41.5) \simeq 8 \times 10^{-6}$ [74]. Since $\Omega_{\mathrm{GW}, 0} \sim(4 / 100)^{1 / 3} 2 \mathcal{A}^{2} / 3 z_{\mathrm{eq}} \sim 7.6 \times 10^{-5} \mathcal{A}^{2}\left(z_{\mathrm{eq}} \sim 3000\right.$ is the redshift at the matter-radiation equality, and the factor $z_{\text {eq }}^{-1}$ reflects $\Omega_{\mathrm{GW}} \propto(1+z) /\left(1+z_{\mathrm{eq}}\right)$ during the matter-dominated era), we obtain $\mathcal{A}^{2} \lesssim 0.1^{\dagger 13}$.

Pulsar timing arrays (PTAs) have also been used to constrain PGWs. Following [72] we use the most stringent upper bound around $f=5.72 \times 10^{-9} \mathrm{~Hz}\left(\sim 4 \times 10^{6} \mathrm{Mpc}^{-1}\right), \Omega_{\mathrm{GW}, 0} \sim$ $(4 / 11)^{1 / 3} 2 \mathcal{A}^{2} / 3 z_{\text {eq }} \lesssim 2 \times 10^{-8}$, which leads to $\mathcal{A}^{2} \lesssim 1.3 \times 10^{-4}$.

Ground-based detectors or PTA experiments constrain PGWs on a relatively limited frequency range, while cosmological methods such as PBHs probe PGWs on a wide range of frequencies, and this is another advantage of our new limits (see Fig. 4).

These upper bounds as a function of $k_{p}$ are shown in Fig. 4 along with the upper bound from PBHs. One may not regard some of weak constraints there as meaningful, because they correspond to (almost) nonlinear tensor perturbations.

As shown in Fig. 15, the upper bounds from PBHs can also be expressed in terms of $\Delta N_{\mathrm{GW}}$

$\dagger 13$ Though not included in our analysis, they also obtained weaker upper bounds on a few frequency ranges other than the one around $\sim 100 \mathrm{~Hz}$. Also, strictly speaking in [74] some power-low spectrum of gravitational waves is assumed in each band, and so their results may not be directly translated into constraints on a narrow peak in the power spectrum we consider. Indeed in [75] an optimal analysis method is discussed to search for a sharp emission line of gravitational waves, which can increase the signal-to-noise ratio by up to a factor of seven. Namely, our comparison here may be crude, but it is sufficient for our purposes. The same applies to the comparison with PTA. 
using (4.23), and also in terms of the current energy density parameter of PGWs, $\Omega_{\mathrm{GW}, 0}$, using

$$
\Omega_{\mathrm{GW}, 0}=\frac{2 \mathcal{A}^{2}}{3 z_{\mathrm{eq}}}\left(\frac{g_{S 0}}{g_{S}\left(T_{\mathrm{in}}\right)}\right)^{1 / 3}
$$

which follows from (4.18) and (4.22). Note that if future experiments reveal the presence of $\Delta N_{\text {eff }}$, then PGWs provide a possible explanation, as well as dark radiation. However, if the value of $\Delta N_{\text {eff }}$ is large, say 0.5 , exceeding the limits shown in Fig. 5, then we may exclude PGWs as a candidate thanks to our PBH bounds ${ }^{\dagger 14}$. This shows an example of how our new limits can provide useful cosmological information.

\section{CONCLUSION}

A novel method using PBH formation to probe primordial gravitational waves is discussed. If the amplitude of tensor perturbations initially on superhorizon scales is very large, substantial scalar perturbations are generated from tensor perturbations. If these induced scalar perturbations are too large, PBHs are overproduced, exceeding existing upper limits on their abundance.

To constrain tensor modes by $\mathrm{PBHs}$ formed by gravitational collapse of radiation overdensities, we have calculated the PDF of the radiation density perturbations, which is in general highly nonGaussian since they are sourced by tensor perturbations. Assuming primordial tensor perturbations are Gaussian, an approximate analytic formula of the PDF was derived, which coincides well with the PDF obtained by a Monte Carlo simulation.

Using this PDF we have constrained a delta-function-type power spectrum of primordial tensor perturbations. Our findings are summarized in Fig. 4 .

$\mathrm{PBH}$ constraints are applicable from comoving scales of $\sim \mathrm{Mpc}$ all the way down to those of $\sim 0.1 \mathrm{~m}$ if we assume the number of $e$-folds during inflation is sixty. The exclusion of an overproduction of smallest PBHs $\left(M_{\mathrm{PBH}} \lesssim 10^{5} \mathrm{~g}\right)$ depends on the assumption that stable Planck mass relics are left over at the end of Hawking evaporation, which behave as cold dark matter (see [76], 24] and references therein). The range of comoving scales corresponding to $M_{\mathrm{PBH}} \lesssim 10^{5} \mathrm{~g}$ is roughly $\lesssim 50 \mathrm{~m}$, namely, the upper bounds from PBHs in this range are based on this assumption. If Planck mass relics are not left over, to what extent an overproduction of PBHs lighter than $10^{5} \mathrm{~g}$ is cosmologically problematic is uncertain. Such an overproduction of smallest PBHs may lead to an early matter-dominated era, during which $\mathrm{PBH}$ binaries are formed and emit gravitational waves, or larger PBHs may form due to merger taking place after the collapse of perturbations of PBHs' density, thereby leaving observable traces [77]. Therefore, in principle one may still exclude such an overproduction of smallest PBHs even without the left over of Planck mass relics to fully validate our upper bounds on smallest scales, though we do not discuss it in detail here.

We have used a perturbative expansion based on small perturbations and therefore one may be worried about the validity of the PDF, shown in Fig. 3, close to the threshold of $\delta_{\text {th }} \simeq 0.4$ we adopted, since this value indicates that further nonlinearities may affect. Very naively, next-order corrections would appear in the fundamental equations which are suppressed by $\sim \mathcal{O}\left(h_{i j}\right) \sim \sqrt{0.4} \sim$

$\dagger 14$ There may be a loophole, however. Logically, if PGWs follow a tremendously non-Gaussian PDF, it may be possible to realize large $\Delta N_{\mathrm{GW}}$ without overproducing $\mathrm{PBHs}$. 
0.6 , and this implies that the upper bounds can be affected by $\sim 60 \%$. Certainly this estimation is very naive anda more careful estimation would be merited. If additional nonlinearities enhance induced scalar perturbations, then our upper bounds would be conservative. To see how further nonlinearities affect our limits, one may write down the next-order correction terms, and then the behaviors of these terms would provide insight. A gradient expansion approach may also be helpful (see e.g. [78] and references therein), which is another perturbative scheme based on the smallness of the ratio of spatial derivatives to time derivatives for perturbations on superhorizon scales. It is valid only on superhorizon scales, but nonlinear perturbations can be treated, relevant to PBH formation. If one compares the amplitude of induced perturbations obtained by a gradient expansion approach and that we have obtained, one would gain insight into how nonlinearities might affect. However, this approach is not perfect either, since it does not allow us to evolve perturbations up to the moment of their horizon reenty, necessary to calculate the probability of PBH formation. Refining our results further would be a formidable task. The present formulation would be acceptable, providing moderately precise and potentially conservative bounds, for our purpose here to propose a novel method to constrain primordial tensor perturbations on small scales from PBHs with detailed calculations for the first time. Let us emphasize that, though upper bounds on scalar perturbations from PBHs have long been known, probably since [79], we have newly found upper bounds from PBHs on tensor perturbations as well.

We have also assumed Gaussianity of primordial tensor perturbations, but PBH constraints on tensor perturbations naturally depend on their statistical properties, determining those of induced density perturbations, just as PBH constraints on scalar perturbations depend on the statistical properties of scalar perturbations [80]. If high- $\sigma$ realizations of tensor perturbations are suppressed (enhanced) in comparison to a Gaussian case, $\mathrm{PBH}$ constraints on tensor perturbations are tighter (weaker).

We have restricted attention to $\mathrm{PBH}$ formation as a result of direct collapse of radiation density perturbations induced by tensor perturbations, but they would also dissipate to induce CMB spectral distortion, and hence constraints on CMB spectral distortion can also be used to probe tensor perturbations. Furthermore, tensor perturbations naturally induce perturbations in the dark matter energy density as well, and if they are sufficiently large, they result in a substantial formation of what are sometimes called ultracompact minihalos, small dark matter halos formed well before the standard structure formation, say $z \sim 1000$. That is, (potential) constraints on them can also be translated into upper bounds on tensor perturbations, which will be explored elsewhere [81].

Lastly, our analysis based on the delta-function spectrum also has implications on constraining other types of tensor power spectra. To see this let us consider the following blue spectrum:

$$
\mathcal{P}_{h}(k)=r \mathcal{P}_{\zeta}\left(k_{\text {ref }}\right)\left(\frac{k}{k_{\text {ref }}}\right)^{n_{T}} \text { for } k<k_{\max },
$$

where $\mathcal{P}_{\zeta}$ is the dimensionless power spectrum of the curvature perturbation, $r$ is the tensorto-scalar ratio, $k_{\text {ref }}$ is some reference wave number and $n_{T}>0$ is the tensor spectral index. If $n_{T}$ is relatively large, say, the upper limit 0.45 obtained below, the amplitude of gravitational waves is mostly determined by the modes with wave number close to $k_{\max }$, and as a result the above spectrum can roughly be regarded as equivalent to a delta-function spectrum (3.47) with $k_{p}=k_{\max }$ and

$$
\mathcal{A}^{2}=\int_{\mathrm{e}^{-1} k_{\max }}^{k_{\max }} r \mathcal{P}_{\zeta}\left(k_{\mathrm{ref}}\right)\left(\frac{k}{k_{\mathrm{ref}}}\right)^{n_{T}} \frac{d k}{k} .
$$


As an illustration, in the following we take $r=0.01, k_{\text {ref }}=0.01 \mathrm{Mpc}^{-1}, \mathcal{P}\left(k_{\text {ref }}\right)=2.2 \times 10^{-9}$ and $k_{\text {max }}=10^{18} \mathrm{Mpc}^{-1}$. Using $\mathcal{A}^{2} \lesssim 0.02$ at around $10^{18} \mathrm{Mpc}^{-1}$ from Fig. 4, we obtain $n_{T} \lesssim 0.45$ from the above $\mathrm{e}^{\dagger 15}$. Here we have neglected the modes with $k<\mathrm{e}^{-1} k_{\max }$, but this probably makes this limit on $n_{T}$ conservative, since contributions of those modes also create density perturbations collapsing to PBHs. Having said that, dedicated calculations for other types of tensor power spectra would be merited.

\section{Appendix A:}

\section{Derivation of evolution equations for induced scalar perturbations}

In this appendix we derive the fundamental equations for scalar perturbations induced by tensor perturbations. First we derive the parts of the equations involving only scalar perturbations, and then we derive the source terms, second order in tensor perturbations. We have also checked the expressions below by a Mathematica package, xPand [82].

\section{Scalar perturbation}

We use the formulation of [68], in which the metric is decomposed as

$$
\begin{gathered}
g_{\mu \nu}=\bar{g}_{\mu \nu}+\delta g_{\mu \nu}, \\
\bar{g}_{00}=-1, \quad \bar{g}_{i 0}=\bar{g}_{0 i}=0, \quad \bar{g}_{i j}=a^{2} \delta_{i j} .
\end{gathered}
$$

The components of the perturbed Ricci tensor are expressed in terms of $\delta g_{\mu \nu}$ as follows $[68]^{\dagger 16}$ :

$$
\begin{aligned}
\delta R_{j k}= & -\frac{1}{2} \delta g_{00, j k}-\left(2 \dot{a}^{2}+a \ddot{a}\right) \delta g_{00} \delta_{j k}-\frac{1}{2} a \dot{a} \dot{\delta} g_{00} \delta_{j k} \\
& +\frac{1}{2 a^{2}}\left(\Delta \delta g_{j k}-\delta g_{i k, i j}-\delta g_{i j, i k}+\delta g_{i i, j k}\right) \\
& -\frac{1}{2} \ddot{\delta} g_{j k}+\frac{\dot{a}}{2 a}\left(\dot{\delta g_{j k}}-\dot{\delta g_{i i}} \delta_{j k}\right)+\frac{\dot{a}^{2}}{a^{2}}\left(-2 \delta g_{j k}+\delta g_{i i} \delta_{j k}\right)+\frac{\dot{a}}{a} \delta g_{i 0, i} \delta_{j k} \\
& +\frac{1}{2}\left(\dot{\delta} g_{k 0, j}+\dot{\delta} g_{j 0, k}\right)+\frac{\dot{a}}{2 a}\left(\delta g_{k 0, j}+\delta g_{j 0, k}\right),
\end{aligned}
$$

$\dagger 15$ The limit on $n_{T}$ is relatively insensitive to the lower bound $k_{\min }$ of the integration of (5.2): we obtain $n_{T} \lesssim 0.47$ for $k_{\min }=2 k_{\max } / 3$, and $n_{T} \lesssim 0.43$ for $k_{\min }=0$.

$\dagger 16$ In [68] the Ricci tensor is defined by

$$
R_{\mu \nu} \equiv \Gamma_{\mu \lambda, \nu}^{\lambda}-\Gamma_{\mu \nu, \lambda}^{\lambda}+\Gamma_{\mu \lambda}^{\kappa} \Gamma_{\nu \kappa}^{\lambda}-\Gamma_{\mu \nu}^{\kappa} \Gamma_{\lambda \kappa}^{\lambda} .
$$

With this definition, the Einstein equations are written as

$$
R_{\mu \nu}-\frac{1}{2} g_{\mu \nu} R=-8 \pi G T_{\mu \nu}
$$

If we adopt another definition of the Ricci tensor, which is minus that of (5.5), then the sign of the right hand side of the above Einstein equations should be flipped. We adopt the former definition in this section following [68], but in the next section we adopt the latter definition. 


$$
\begin{aligned}
\delta R_{0 j}=\delta R_{j 0}= & \frac{\dot{a}}{a} \delta g_{00, j}+\frac{1}{2 a^{2}}\left(\Delta \delta g_{j 0}-\delta g_{i 0, j i}\right)-\left(\frac{\ddot{a}}{a}+\frac{2 \dot{a}^{2}}{a^{2}}\right) \delta g_{j 0} \\
& +\frac{1}{2} \frac{\partial}{\partial t}\left[\frac{1}{a^{2}}\left(\delta g_{k k, j}-\delta g_{k j, k}\right)\right] \\
\delta R_{00}= & \frac{1}{2 a^{2}} \Delta \delta g_{00}+\frac{3 \dot{a}}{2 a} \dot{\delta g_{00}}-\frac{1}{a^{2}} \dot{\delta} g_{i 0, i} \\
& +\frac{1}{2 a^{2}}\left[\ddot{\delta g} g_{i i}-\frac{2 \dot{a}}{a} \dot{\delta} g_{i i}+2\left(\frac{\dot{a}^{2}}{a^{2}}-\frac{\ddot{a}}{a}\right) \delta g_{i i}\right] .
\end{aligned}
$$

The components of the Ricci tensor with mixed indices are expressed in terms of those with doubly covariant indices as follows:

$$
\begin{gathered}
\delta R_{0}^{0}=-3 \frac{\ddot{a}}{a} \delta g_{00}-\delta R_{00}, \\
\delta R_{i}^{0}=-\delta R_{0 i}-a^{-2}\left(2 \dot{a}^{2}+a \ddot{a}\right) \delta g_{i 0}, \\
\delta R_{j}^{i}=a^{-2}\left(2 H^{2}+\frac{\ddot{a}}{a}\right) \delta g_{i j}+\frac{1}{a^{2}} \delta R_{i j} .
\end{gathered}
$$

Using these, the Ricci scalar can be calculated as

$$
\begin{aligned}
a^{2} \delta R= & -3 a \dot{a} \dot{\delta} g_{00}-6\left(\dot{a}^{2}+a \ddot{a}\right) \delta g_{00}-\Delta \delta g_{00}+2 \dot{\delta g_{i 0, i}}+4 H \delta g_{i 0, i} \\
& -\ddot{\delta} g_{i j}+\frac{2}{3 a^{2}} \Delta \delta g_{i i}+2\left(H^{2}+\frac{\ddot{a}}{a}\right) \delta g_{i i} .
\end{aligned}
$$

In our notation of (3.3),

$$
\delta g_{00}=-2 \Phi, \quad \delta g_{i 0}=a B_{, i}, \quad \delta g_{i i}=-6 a^{2} \Psi .
$$

The time-time component of the Einstein equations is

$$
\frac{a^{2}}{2} G_{0}^{0}=\Delta \Psi-3 \mathcal{H}\left(\Psi^{\prime}+\mathcal{H}^{2} \Phi\right)-\mathcal{H} \Delta B=\frac{a^{2}}{2} 8 \pi G \delta \rho,
$$

which recovers the parts of (3.8) involving scalar perturbations. The time-space component is

$$
G_{i}^{0}=R_{i}^{0}=-\delta R_{0 i}-a^{-2}\left(2 \dot{a}^{2}+a \ddot{a}\right) \delta g_{i 0}=2 \dot{\Psi}_{, i}+2 H \Phi_{, i} .
$$

So $a G_{i}^{0} / 2=0$ partially recovers (3.9). The space-space components are

$$
\delta G_{j}^{i}=a^{-2}\left(2 H^{2}+\frac{\ddot{a}}{a}\right) \delta g_{i j}+\frac{1}{a^{2}} \delta R_{i j}-\frac{1}{2} \delta R \delta_{i j},
$$

and this is written in the form $\delta G_{j}^{i}=G_{1} \delta_{i j}+G_{2, i j}$, where

$$
\begin{gathered}
-\frac{a^{2}}{2} G_{1}=\Psi^{\prime \prime}+\mathcal{H}(2 \Psi+\Phi)^{\prime}+\left(2 \mathcal{H}^{\prime}+\mathcal{H}^{2}\right) \Phi+\frac{1}{2} \Delta\left(\Phi-\Psi+B^{\prime}+2 \mathcal{H} B\right), \\
a^{2} G_{2}=\Phi-\Psi+B^{\prime}+2 \mathcal{H} B .
\end{gathered}
$$

Then, $-a^{2} G_{1} / 2=a^{2} 8 \pi G \delta p / 2$ partially recovers (3.10), and $a^{2} G_{2, i j}=0$ partially recovers (3.11). Also, (3.21) and (3.22) without the source term can be derived from (5.1.49) and (5.1.48) of $[68]^{\dagger 17}$.

$\dagger 17$ One can also confirm that, dropping the source terms originating from tensor perturbations, Eqs. (3.8)(3.11), (3.21) and (3.22) reduce to Eqs. (A.98)-(A.103) of [69]. 


\section{Tensor perturbation}

Let us consider the following metric

$$
d s^{2}=a^{2}(\eta)\left[-d \eta^{2}+a^{2}\left(\delta_{i j}+\tilde{h}_{i j}\right) d x^{i} d x^{j}\right],
$$

where $\tilde{h}_{i j}$ is two times $h_{i j}$ in (3.3) and is introduced here for simplicity. We decompose the metric (and other tensors below) as $g_{i j}=\bar{g}_{i j}+\delta g_{i j}+\delta^{2} g_{i j}$, with $\bar{g}_{i j}=a^{2} \delta_{i j}, \delta g_{i j}=a^{2} \tilde{h}_{i j}, \delta^{2} g_{i j}=0$. Then, $\bar{g}^{i j}=a^{-2} \delta_{i j}, \delta g_{i j}=-a^{2} \tilde{h}_{i j}, \delta^{2} g^{i j}=\tilde{h}^{i k} \tilde{h}_{k}^{j}$. The indices of $\tilde{h}_{i j}$ are raised and lowered by $\delta_{i j}$. The nonvanishing components of the Christoffel symbol are

$$
\begin{gathered}
\bar{\Gamma}_{00}^{0}=\mathcal{H}, \quad \bar{\Gamma}_{i j}^{0}=\mathcal{H} \delta_{i j}, \quad \delta \Gamma_{i j}^{0}=\frac{1}{2}\left(\tilde{h}_{i j}^{\prime}+2 \mathcal{H} \tilde{h}_{i j}\right), \\
\bar{\Gamma}_{j 0}^{i}=\mathcal{H} \delta_{i j}, \quad \delta \Gamma_{j 0}^{i}=\frac{1}{2} \tilde{h}_{j}^{i^{\prime}}, \quad \delta^{2} \Gamma_{j 0}^{i}=-\frac{1}{2} \tilde{h}^{i k} \tilde{h}_{k j}^{\prime}, \\
\delta \Gamma_{j k}^{i}=\frac{1}{2}\left(\tilde{h}_{i j, k}+\tilde{h}_{i k, j}-\tilde{h}_{j k, i}\right), \quad \delta^{2} \Gamma_{j k}^{i}=\frac{1}{2} \tilde{h}^{i l}\left(\tilde{h}_{l j, k}+\tilde{h}_{l k, j}-\tilde{h}_{j k, l}\right) .
\end{gathered}
$$

The components of the Ricci tensor are

$$
\begin{gathered}
\delta^{2} R_{00}=\frac{1}{2} \tilde{h}^{i j} \tilde{h}_{i j}^{\prime \prime}+\frac{1}{4} \tilde{h}^{i j^{\prime}} \tilde{h}_{i j}^{\prime}+\frac{1}{2} \mathcal{H} \tilde{h}^{i j} \tilde{h}_{i j}^{\prime}, \\
\delta^{2} R_{i 0}=\frac{1}{4} \tilde{h}^{j k^{\prime}} \tilde{h}_{j k, i}+\frac{1}{2} \tilde{h}^{j k} \tilde{h}_{j k, i}^{\prime}-\frac{1}{2} \tilde{h}^{j k} \tilde{h}_{i j, k}^{\prime}, \\
\bar{R}_{i j}=\left(\mathcal{H}^{\prime}+2 \mathcal{H}^{2}\right) \delta_{i j}, \\
\delta R_{i j}=\frac{1}{2} \tilde{h}_{i j}^{\prime \prime}+\mathcal{H} \tilde{h}_{i j}^{\prime}+\left(\mathcal{H}^{\prime}+2 \mathcal{H}^{2}\right) \tilde{h}_{i j}-\frac{1}{2} \Delta \tilde{h}_{i j}, \\
\delta^{2} R_{i j}=-\frac{\mathcal{H}}{2} \tilde{h}^{k l} \tilde{h}_{k l}^{\prime} \delta_{i j}-\frac{1}{2} \tilde{h}_{i}^{k \prime} \tilde{h}_{k j}^{\prime}+\frac{1}{2} \tilde{h}^{k l}\left(\tilde{h}_{i j, k l}-\tilde{h}_{i k, j l}-\tilde{h}_{j k, i l}\right)+\frac{1}{2} \tilde{h}^{k l} \tilde{h}_{k l, i j} \\
+\frac{1}{4} \tilde{h}_{, i}^{k l} \tilde{h}_{k l, j}+\frac{1}{2} \tilde{h}_{i}^{k, l} \tilde{h}_{j k, l}-\frac{1}{2} \tilde{h}_{i}^{k, l} \tilde{h}_{j l, k} .
\end{gathered}
$$

The components of the Ricci tensor with mixed indices are given by

$$
\begin{gathered}
\delta^{2} R_{0}^{0}=-a^{-2} \delta^{2} R_{00}, \quad \delta^{2} R_{i}^{0}=-a^{-2} \delta^{2} R_{0 i}, \\
\delta^{2} R_{j}^{i}=\delta^{2} g^{i k} \bar{R}_{k j}+\delta g^{i k} \delta R_{k j}+a^{-2} \delta^{2} R_{i j} .
\end{gathered}
$$

The Ricci scalar can be written as

$$
\delta^{2} R=-a^{-2} \delta^{2} R_{00}+a^{-2} \delta^{2} R_{i i}+\delta g^{i j} \delta R_{i j}+\delta^{2} g^{i j} \bar{R}_{i j},
$$

which leads to

$$
a^{2} \delta^{2} R=-\tilde{h}^{i j} \tilde{h}_{i j}^{\prime \prime}-\frac{3}{4} \tilde{h}^{i j^{\prime}} \tilde{h}_{i j}^{\prime}-3 \mathcal{H} \tilde{h}^{i j} \tilde{h}_{i j}^{\prime}+\tilde{h}^{i j} \Delta \tilde{h}_{i j}+\frac{3}{4} \tilde{h}^{i j, k} \tilde{h}_{i j, k}-\frac{1}{2} \tilde{h}^{i j, k} \tilde{h}_{i k, j} .
$$

The components of the Einstein tensor are

$$
\begin{gathered}
-\frac{a^{2}}{2} \delta^{2} G_{0}^{0}=S_{1}, \\
\delta^{2} G_{0}^{i}=-\delta^{2} G_{i}^{0}=a^{-2} \delta^{2} R_{i 0}=\frac{2 S_{i}}{a^{2}} \\
a^{2} \delta^{2} G_{j}^{i}=a^{2}\left(\delta^{2} g^{i k} \bar{R}_{k j}+\delta g^{i k} \delta R_{k j}+a^{-2} \delta^{2} R_{i j}\right)=2 S_{3} \delta_{i j}+2 S_{i j} .
\end{gathered}
$$


Eqs. (5.15) and (5.33) recover (3.8) (see footnott†16). Also, Eqs. (5.16) and (5.34) recover (3.9) ${ }^{\dagger 18}$. Let us decompose $S_{i j}$ as $S_{i j}=S_{4} \delta_{i j}+S_{5, i j}+\cdots$, where $\cdots$ is to contain vector and tensor parts, which are irrelevant here. From this, we find $\Delta S_{i}^{i}=3 \Delta S_{4}+\Delta^{2} S_{5}$ and $S^{i j}{ }_{i j}=\Delta S_{4}+\Delta^{2} S_{5}$, which lead to (3.15) and (3.16). Then we find (3.10) and (3.11) from (5.18), (5.19) and (5.35).

The second-order parts of the divergence of the energy momentum tensor are

$$
\delta^{2} T_{\nu ; \mu}^{\mu}=\delta^{2} \Gamma_{\mu \lambda}^{\mu} \bar{T}_{\nu}^{\lambda}-\delta^{2} \Gamma_{\mu \nu}^{\lambda} \bar{T}_{\lambda}^{\mu},
$$

which is nonzero when $\nu=0$ :

$$
\delta^{2} T_{0 ; \mu}^{\mu}=2(\rho+p) h^{i j} h_{i j}^{\prime} .
$$

The negative of this gives the source term of (3.21).

\section{Appendix B: Derivation of the source term in Fourier space}

In this appendix we derive (3.43) and (3.44). First, note that the Fourier components of $h^{i j} h_{i j}$ can be expressed as

$$
\left(h^{i j} h_{i j}\right)(\eta, \boldsymbol{k})=\int \frac{d^{3} \boldsymbol{k}^{\prime}}{(2 \pi)^{3 / 2}} \sum_{r s} h^{r}\left(\boldsymbol{k}^{\prime}\right) h^{s}\left(\boldsymbol{k}-\boldsymbol{k}^{\prime}\right) D\left(\eta, k^{\prime}\right) E_{r s i j}^{i j}\left(\boldsymbol{k}, \boldsymbol{k}^{\prime}\right) D\left(\eta,\left|\boldsymbol{k}-\boldsymbol{k}^{\prime}\right|\right) .
$$

Similarly, the source can be written as

$$
S(\eta, \boldsymbol{k})=\int \frac{d^{3} \boldsymbol{k}^{\prime}}{(2 \pi)^{3 / 2}} \sum_{r s} h^{r}\left(\boldsymbol{k}^{\prime}\right) h^{s}\left(\boldsymbol{k}-\boldsymbol{k}^{\prime}\right) D\left(\eta, k^{\prime}\right)(\cdots) D\left(\eta,\left|\boldsymbol{k}-\boldsymbol{k}^{\prime}\right|\right) .
$$

In the following, let us consider the contribution of each term in (3.26) to $(\cdots)$ of the above expression. The contribution of the term $\partial_{j} h_{i k} \partial^{k} h^{i j}=\partial_{j} \partial^{k}\left(h_{i k} h^{i j}\right)$ in (3.12) to ( ...), indicated after the arrow in the equation below (the arrows elsewhere should be understood similarly), is

$$
\partial_{j} h_{i k} \partial^{k} h^{i j}=\partial_{j} \partial^{k}\left(h_{i k} h^{i j}\right) \quad \rightarrow \quad-k^{2} E_{1}^{r s} .
$$

Similarly,

$$
\partial_{k} h_{i j} \partial^{k} h^{i j}=\frac{1}{2} \partial_{k} \partial^{k}\left(h_{i j} h^{i j}\right)-\left(\Delta h_{i j}\right) h^{i j} \quad \rightarrow \quad-\frac{1}{2} k^{2} E_{2}^{r s}+k^{\prime 2} E_{2}^{r s} .
$$

So the contribution of $S_{1}$ is

$$
S_{1} \quad \rightarrow \quad\left(-\frac{1}{4} \overleftarrow{\partial_{\eta}} \partial_{\eta}+\partial_{\eta}^{2}-\frac{3}{8} k^{2}+\frac{3}{4} k^{\prime 2}\right) E_{2}^{r s}+\frac{k^{2}}{2} E_{1}^{r s}
$$

where $\overleftarrow{\partial_{\eta}}$ is supposed to differentiate only $D\left(\eta, k^{\prime}\right)$ of Eq.(15.39) in the left. Likewise, the contribution of $S_{3}$ is

$$
S_{3} \quad \rightarrow \quad\left(\frac{3}{4} \overleftarrow{\partial_{\eta}} \partial_{\eta}+\frac{3}{8} k^{2}-\frac{3}{4} k^{\prime 2}\right) E_{2}^{r s}-\frac{k^{2}}{2} E_{1}^{r s}
$$

To obtain the contribution of $\hat{k}^{i} \hat{k}^{j} S_{i j}$, let us rewrite $S_{i j}$ as follows:

$$
\begin{array}{r}
S_{i j}=-h_{i}{ }^{k^{\prime}} h_{j k}^{\prime}+\partial_{k} \partial_{l}\left(h^{k l} h_{i j}\right)-\partial_{l}\left(h^{k l} \partial_{i} h_{j k}\right)-(i \leftrightarrow j)-\partial_{k} \partial^{l}\left(h_{j l} h_{i}{ }^{k}\right) \\
+\partial_{l} h_{j k} \partial^{l} h_{i}{ }^{k}+\frac{1}{2} \partial_{i} \partial_{j}\left(h^{k l} h_{k l}\right)-\frac{1}{2} \partial_{i} h^{k l} \partial_{j} h_{k l} .
\end{array}
$$

$\dagger 18$ The indices " 0 " indicate $t$ in the previous subsection, while those indicate the conformal time $\eta$ in this subsection, and they are related by $G_{i}^{t}=a G_{i}^{\eta}$. 
Then, the contribution is

$$
\begin{array}{r}
S_{i j} \hat{k}^{i} \hat{k}^{j} \rightarrow \quad-\overleftarrow{\partial_{\eta}} \partial_{\eta} E_{i j k}{ }_{j k} \hat{k}^{i} \hat{k}^{j}-k_{k} k_{l} E^{k l}{ }_{i j} \hat{k}^{i} \hat{k}^{j}+2 k_{l}\left(k_{i}-k_{i}^{\prime}\right) E^{k l}{ }_{j k} \hat{k}^{i} \hat{k}^{j} \\
+k_{k} k^{l} E_{j l i}{ }^{k} \hat{k}^{i} \hat{k}^{j}-k_{l}^{\prime}\left(k^{l}-k^{\prime l}\right) E_{j k i}{ }^{k} \hat{k}^{i} \hat{k}^{j}-\frac{1}{2} k_{i} k_{j} \hat{k}^{i} \hat{k}^{j} E^{k l}{ }_{k l} \\
+\frac{1}{2} k_{i}^{\prime}\left(k_{j}-k_{j}^{\prime}\right) E^{k l}{ }_{k l} \hat{k}^{i} \hat{k}^{j} \\
=\left(-\overleftarrow{\partial_{\eta}} \partial_{\eta}+2 k^{2}-3 k k^{\prime} \mu+k^{\prime 2}\right) E_{1}^{r s}+\frac{1}{2}\left(k^{\prime} \mu\left(k-k^{\prime} \mu\right)-k^{2}\right) E_{2}^{r s} .
\end{array}
$$

The collection of all the contributions yields

$$
\begin{aligned}
& S \rightarrow\left\{\overleftarrow{\partial_{\eta}} \partial_{\eta}-\frac{1}{2}\left(3-c_{\mathrm{s}}^{2}\right) k^{2}+3 k k^{\prime} \mu-k^{\prime 2}\right\} E_{1}^{r s}+ \\
& \left\{-\frac{1}{4}\left(3+c_{\mathrm{s}}^{2}\right) \overleftarrow{\partial_{\eta}} \partial_{\eta}+c_{\mathrm{s}}^{2} \partial_{\eta}^{2}+2 c_{\mathrm{s}}^{2} \mathcal{H} \partial_{\eta}+\frac{1}{8}\left(1-3 c_{\mathrm{s}}^{2}\right) k^{2}-\frac{1}{2} k^{\prime} \mu\left(k-k^{\prime} \mu\right)+\frac{3}{4}\left(1+c_{\mathrm{s}}^{2}\right) k^{\prime 2}\right\} E_{2}^{r s},
\end{aligned}
$$

from which (3.43) and (3.44) can be read off.

\section{Appendix C: PDF of induced radiation density perturbations}

In this paper a delta-function-type tensor spectrum is assumed (see (3.47)), but since it cannot be treated in numerical calculations adopting discretization in Fourier space, the power spectrum is instead approximated by the following top-hat spectrum here:

$$
\mathcal{P}_{h}(k)=\mathcal{A}^{2} \epsilon^{-1}\left(k_{p}\left[1-\frac{\epsilon}{2}\right]<k<k_{p}\left[1+\frac{\epsilon}{2}\right]\right), \quad 0 \text { (otherwise). }
$$

In this appendix, we set $\mathcal{A}=1$, and $\epsilon$ is chosen to be sufficiently small, as presented shortly.

Let us decompose the Fourier components of $h^{r}(\boldsymbol{k})$ as follows:

$$
h^{r}(\boldsymbol{k})=a^{r}(\boldsymbol{k})+i b^{r}(\boldsymbol{k}),
$$

where $a^{r}$ and $b^{r}$ are real Gaussian random variables satisfying

$$
a^{+}(-\boldsymbol{k})=a^{+}(\boldsymbol{k}), \quad b^{+}(-\boldsymbol{k})=-b^{+}(\boldsymbol{k}), \quad a^{\times}(-\boldsymbol{k})=-a^{\times}(\boldsymbol{k}), \quad b^{\times}(-\boldsymbol{k})=b^{\times}(\boldsymbol{k})
$$

to ensure the reality of $h_{i j}(\eta, \boldsymbol{x})$ (note that $e_{i j}^{\times}(-\boldsymbol{k})=-e_{i j}^{\times}(\boldsymbol{k})$ as well as $e_{i j}^{+}(-\boldsymbol{k})=e_{i j}^{+}(\boldsymbol{k})$ following the definitions of the polarization tensors we adopt). We consider a spherical shell in the Fourier space whose radius is $k_{p}$ and whose thickness is $\epsilon k_{p}$, as is depicted in Fig. 6. Let us denote the grid points in this spherical shell by $\boldsymbol{k}_{i}$, where $i$ is a natural number. Each of these grid points is associated with two complex numbers $h^{r}\left(\boldsymbol{k}_{i}\right)=a^{r}\left(\boldsymbol{k}_{i}\right)+i b^{r}\left(\boldsymbol{k}_{i}\right),(r=+, \times)$ (satisfying $\left.h^{+}\left(-\boldsymbol{k}_{i}\right)=h^{+}\left(\boldsymbol{k}_{i}\right)^{*}, h^{\times}\left(-\boldsymbol{k}_{i}\right)=-h^{\times}\left(\boldsymbol{k}_{i}\right)^{*}\right)$, where the dispersion of both $a^{r}$ and $b^{r}$ is

$$
\sigma^{2}=\frac{\pi^{2}}{k_{p}^{3}} d k^{-3} \epsilon^{-1}
$$

with $d k$ denoting the interval between two neighboring grid points in the Fourier space. Then, from (3.48), $\delta_{r}(\eta, \boldsymbol{x}=0, R)$ for a specific realization of $\left\{h^{r}\left(\boldsymbol{k}_{i}\right)\right\}$ is calculated by

$$
\delta_{r}(\eta, \boldsymbol{x}=0, R)=\frac{1+c_{\mathrm{s}}^{2}}{c_{\mathrm{s}}^{2} \mathcal{H}} \frac{(d k)^{6}}{(2 \pi)^{3}}\left\{\sum_{r, s} \sum_{\boldsymbol{k}_{i}, \boldsymbol{k}_{j} \in S} W\left(\left|\boldsymbol{k}_{i}+\boldsymbol{k}_{j}\right| R\right) h^{r}\left(\boldsymbol{k}_{i}\right) h^{s}\left(\boldsymbol{k}_{j}\right) F_{r s}\left(\eta, \boldsymbol{k}_{i}+\boldsymbol{k}_{j}, \boldsymbol{k}_{i}\right)\right\},
$$

where $S$ denotes the set comprised of the grid points inside the spherical shell. As mentioned in the main text, we set $\eta=R=\left(0.7 k_{p}\right)^{-1}$. When one is interested in the power spectrum, (3.40) 


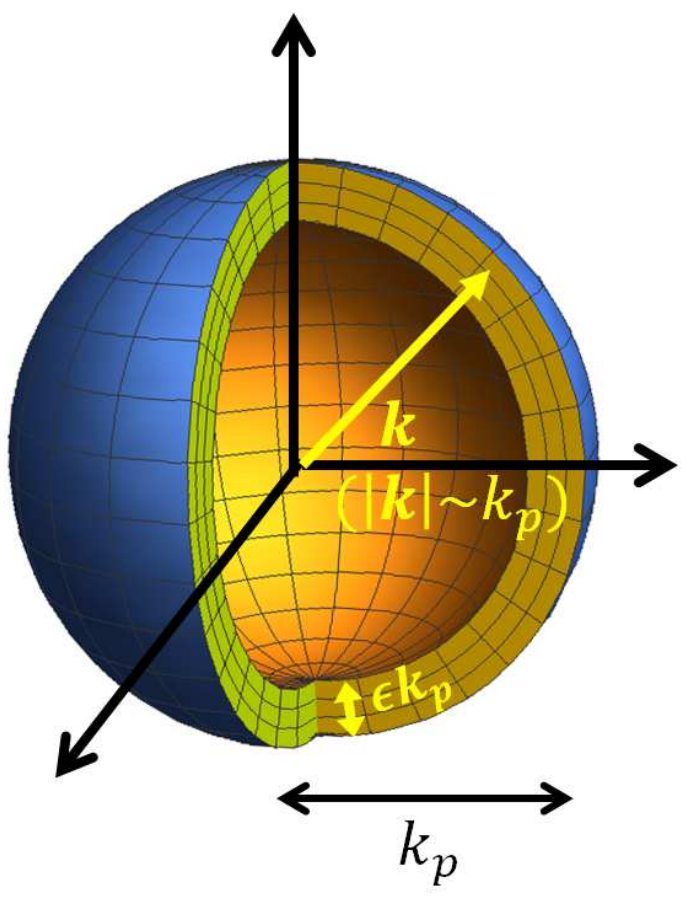

FIG. 6: An illustration of the spherical shell in the Fourier space considered in this appendix.

and (3.41) can be used due to isotropy, but in simulations their components, including nonzero cross terms, for each combination of wave vectors have to be explicitly calculated using

$$
\begin{gathered}
e_{i j}^{+}(\hat{k})=\left(\begin{array}{ccc}
1 & 0 & 0 \\
0 & -1 & 0 \\
0 & 0 & 0
\end{array}\right)\left(\left|\hat{k}_{3}\right|=1\right), \quad\left(\begin{array}{ccc}
\frac{\hat{k}_{3}^{2}-\hat{k}_{3}^{4}-\hat{k}_{2}^{2}\left(1+\hat{k}_{3}^{2}\right)}{-1+\hat{k}_{3}^{2}} & \frac{\hat{k}_{1} \hat{k}_{2}\left(1+\hat{k}_{3}^{2}\right)}{-1+\hat{k}_{3}^{2}} & \hat{k}_{1} \hat{k}_{3} \\
\frac{\hat{k}_{1} \hat{k}_{2}\left(1+\hat{k}_{3}^{2}\right)}{-1+\hat{k}_{3}^{2}} & \frac{-1+\hat{k}_{3}^{2}+\hat{k}_{2}^{2}\left(1+\hat{k}_{3}^{2}\right)}{-1+\hat{k}_{3}^{2}} & \hat{k}_{2} \hat{k}_{3} \\
\hat{k}_{1} \hat{k}_{3} & \hat{k}_{2} \hat{k}_{3} & -1+\hat{k}_{3}^{2}
\end{array}\right)\left(\left|\hat{k}_{3}\right| \neq 1\right), \\
e_{i j}^{\times}(\hat{k})= \pm\left(\begin{array}{lll}
0 & 1 & 0 \\
1 & 0 & 0 \\
0 & 0 & 0
\end{array}\right)\left(\hat{k}_{3}= \pm 1\right), \quad\left(\begin{array}{ccc}
-\frac{2 \hat{k}_{1} \hat{k}_{2} \hat{k}_{3}}{-1+\hat{k}_{3}^{2}} & -\frac{\hat{k}_{3}\left(-1+2 \hat{k}_{2}^{2}+\hat{k}_{3}^{2}\right)}{-1+\hat{k}_{3}^{2}} & -\hat{k}_{2} \\
-\frac{\hat{k}_{3}\left(-1+2 \hat{k}_{2}^{2}+\hat{k}_{3}^{2}\right)}{-1+\hat{k}_{3}^{2}} & \frac{2 \hat{k}_{1} \hat{k}_{2} \hat{k}_{3}}{-1+\hat{k}_{3}^{2}} & \hat{k}_{1} \\
-\hat{k}_{2} & \hat{k}_{1} & 0
\end{array}\right)\left(\left|\hat{k}_{3}\right| \neq 1\right) .
\end{gathered}
$$

Using some of its symmetry properties, (5.51) can be simplified as follows. Let us denote by $S / 2$ the set of the grid points inside the upper half of the spherical shell. More precisely, the set $S / 2$ is made up of the grid points $\left\{\boldsymbol{k}_{i}\right\}$ in the spherical shell with $\left(\boldsymbol{k}_{i}\right)_{z}>0$, those with $\left(\boldsymbol{k}_{i}\right)_{z}=0$ and $\left(\boldsymbol{k}_{i}\right)_{y}>0$, and also those with $\left(\boldsymbol{k}_{i}\right)_{z}=\left(\boldsymbol{k}_{i}\right)_{y}=0$ and $\left(\boldsymbol{k}_{i}\right)_{x}>0$. Then, the inside of the brace 
of (5.51) can be rewritten as

$$
\begin{gathered}
\sum_{r, s} \sum_{\boldsymbol{k}_{i}, \boldsymbol{k}_{j} \in S / 2}\left[W\left(\left|\boldsymbol{k}_{i}+\boldsymbol{k}_{j}\right|\right)\left\{h^{r}\left(\boldsymbol{k}_{i}\right) h^{s}\left(\boldsymbol{k}_{j}\right) F_{r s}\left(\eta, \boldsymbol{k}_{i}+\boldsymbol{k}_{j}, \boldsymbol{k}_{i}\right)+h^{r}\left(-\boldsymbol{k}_{i}\right) h^{s}\left(-\boldsymbol{k}_{j}\right) F_{r s}\left(\eta,-\boldsymbol{k}_{i}-\boldsymbol{k}_{j},-\boldsymbol{k}_{i}\right)\right\}\right. \\
\left.+W\left(\left|\boldsymbol{k}_{i}-\boldsymbol{k}_{j}\right|\right)\left\{h^{r}\left(-\boldsymbol{k}_{i}\right) h^{s}\left(\boldsymbol{k}_{j}\right) F_{r s}\left(\eta,-\boldsymbol{k}_{i}+\boldsymbol{k}_{j},-\boldsymbol{k}_{i}\right)+h^{r}\left(\boldsymbol{k}_{i}\right) h^{s}\left(-\boldsymbol{k}_{j}\right) F_{r s}\left(\eta, \boldsymbol{k}_{i}-\boldsymbol{k}_{j}, \boldsymbol{k}_{i}\right)\right\}\right] \\
=\sum_{r, s} \sum_{\boldsymbol{k}_{i}, \boldsymbol{k}_{j} \in S / 2}\left[W\left(\left|\boldsymbol{k}_{i}+\boldsymbol{k}_{j}\right|\right)\left\{h^{r}\left(\boldsymbol{k}_{i}\right) h^{s}\left(\boldsymbol{k}_{j}\right)+h^{r}\left(\boldsymbol{k}_{i}\right)^{*} h^{s}\left(\boldsymbol{k}_{j}\right)^{*}\right\} F_{r s}\left(\eta, \boldsymbol{k}_{i}+\boldsymbol{k}_{j}, \boldsymbol{k}_{i}\right)\right. \\
\left.+W\left(\left|\boldsymbol{k}_{i}-\boldsymbol{k}_{j}\right|\right) \epsilon_{s}\left\{h^{r}\left(\boldsymbol{k}_{i}\right)^{*} h^{s}\left(\boldsymbol{k}_{j}\right)+h^{r}\left(\boldsymbol{k}_{i}\right) h^{s}\left(\boldsymbol{k}_{j}\right)^{*}\right\} F_{r s}\left(\eta, \boldsymbol{k}_{i}-\boldsymbol{k}_{j}, \boldsymbol{k}_{i}\right)\right] \\
=\sum_{r, s} \sum_{\boldsymbol{k}_{i}, \boldsymbol{k}_{j} \in S / 2}\left[2 W\left(\left|\boldsymbol{k}_{i}+\boldsymbol{k}_{j}\right|\right)\left\{a^{r}\left(\boldsymbol{k}_{i}\right) a^{s}\left(\boldsymbol{k}_{j}\right)-b^{r}\left(\boldsymbol{k}_{i}\right) b^{s}\left(\boldsymbol{k}_{j}\right)\right\} F_{r s}\left(\eta, \boldsymbol{k}_{i}+\boldsymbol{k}_{j}, \boldsymbol{k}_{i}\right)\right. \\
\left.+2 \epsilon_{s} W\left(\left|\boldsymbol{k}_{i}-\boldsymbol{k}_{j}\right|\right)\left\{a^{r}\left(\boldsymbol{k}_{i}\right) a^{s}\left(\boldsymbol{k}_{j}\right)+b^{r}\left(\boldsymbol{k}_{i}\right) b^{s}\left(\boldsymbol{k}_{j}\right)\right\} F_{r s}\left(\eta, \boldsymbol{k}_{i}-\boldsymbol{k}_{j}, \boldsymbol{k}_{i}\right)\right]
\end{gathered}
$$

where we have used $h^{r}\left(-\boldsymbol{k}_{i}\right)=\epsilon_{r} h^{r}\left(\boldsymbol{k}_{i}\right)^{*}\left(\epsilon_{+}=1, \epsilon_{\times}-1\right)$ and $F_{r s}\left(\eta,-\boldsymbol{k},-\boldsymbol{k}^{\prime}\right)=\epsilon_{r} \epsilon_{s} F_{r s}\left(\eta, \boldsymbol{k}, \boldsymbol{k}^{\prime}\right)$. This has explicitly proven that $\delta_{r}$ is real, as it should. Let us label the grid points in $S / 2$ by $1,2, \cdots, N$, then introducing

$$
\begin{aligned}
\boldsymbol{a}^{t} & =\sigma^{-1}\left(a^{+}\left(\boldsymbol{k}_{1}\right), a^{+}\left(\boldsymbol{k}_{2}\right), \cdots, a^{+}\left(\boldsymbol{k}_{N}\right), a^{\times}\left(\boldsymbol{k}_{1}\right), a^{\times}\left(\boldsymbol{k}_{2}\right), \cdots, a^{\times}\left(\boldsymbol{k}_{N}\right)\right), \\
\boldsymbol{b}^{t} & =\sigma^{-1}\left(b^{+}\left(\boldsymbol{k}_{1}\right), b^{+}\left(\boldsymbol{k}_{2}\right), \cdots, b^{+}\left(\boldsymbol{k}_{N}\right), b^{\times}\left(\boldsymbol{k}_{1}\right), b^{\times}\left(\boldsymbol{k}_{2}\right), \cdots, b^{\times}\left(\boldsymbol{k}_{N}\right)\right),
\end{aligned}
$$

and using (5.54) we can rewrite (5.51) as

$$
\delta_{r}(\eta, \boldsymbol{x}=\mathbf{0}, R)=\frac{1+c_{s}^{2}}{c_{s}^{2} \mathcal{H}} \frac{d k^{3}}{8 \pi \epsilon k_{p}^{3}}\left\{\boldsymbol{a}^{t} \boldsymbol{M}^{a} \boldsymbol{a}+\boldsymbol{b}^{t} \boldsymbol{M}^{b} \boldsymbol{b}\right\},
$$

where

$$
\begin{aligned}
& \boldsymbol{M}^{a} \equiv\left(\begin{array}{ll}
\boldsymbol{M}_{++}^{a} & \boldsymbol{M}_{+\times}^{a} \\
\boldsymbol{M}_{\times+}^{a} & \boldsymbol{M}_{\times \times}^{a}
\end{array}\right), \quad \boldsymbol{M}^{b} \equiv\left(\begin{array}{ll}
\boldsymbol{M}_{++}^{b} & \boldsymbol{M}_{+\times}^{b} \\
\boldsymbol{M}_{\times+}^{b} & \boldsymbol{M}_{\times \times}^{b}
\end{array}\right), \\
& \left(\boldsymbol{M}_{r s}^{a}\right)_{i j}=\left(\boldsymbol{M}_{r s}^{1}\right)_{i j}+\left(\boldsymbol{M}_{r s}^{2}\right)_{i j}, \\
& \left(\boldsymbol{M}_{r s}^{b}\right)_{i j}=-\left(\boldsymbol{M}_{r s}^{1}\right)_{i j}+\left(\boldsymbol{M}_{r s}^{2}\right)_{i j}, \\
& \left(\boldsymbol{M}_{r s}^{1}\right)_{i j}=2 W\left(\left|\boldsymbol{k}_{i}+\boldsymbol{k}_{j}\right|\right) F_{r s}\left(\eta, \boldsymbol{k}_{i}+\boldsymbol{k}_{j}, \boldsymbol{k}_{i}\right), \\
& \left(\boldsymbol{M}_{r s}^{2}\right)_{i j}=2 \epsilon_{s} W\left(\left|\boldsymbol{k}_{i}-\boldsymbol{k}_{j}\right|\right) F_{r s}\left(\eta, \boldsymbol{k}_{i}-\boldsymbol{k}_{j}, \boldsymbol{k}_{i}\right) .
\end{aligned}
$$

Noting $F_{r s}\left(\eta, \boldsymbol{k}_{i}+\boldsymbol{k}_{j}, \boldsymbol{k}_{i}\right)=F_{s r}\left(\eta, \boldsymbol{k}_{j}+\boldsymbol{k}_{i}, \boldsymbol{k}_{j}\right)$ and $\epsilon_{s} F_{r s}\left(\eta, \boldsymbol{k}_{i}-\boldsymbol{k}_{j}, \boldsymbol{k}_{i}\right)=\epsilon_{r} F_{s r}\left(\eta, \boldsymbol{k}_{j}-\boldsymbol{k}_{i}, \boldsymbol{k}_{j}\right)$, one can confirm that $\boldsymbol{M}^{a}$ and $\boldsymbol{M}^{b}$ are symmetric matrices. So by diagonalizing $\boldsymbol{M}^{a}$ and $\boldsymbol{M}^{b}($ (5.57) can be further rewritten as

$$
\delta_{r}=a_{0} \sum_{i=1}^{2 N} a_{i} x_{i}^{2},
$$

where $x_{1}, x_{2}, \cdots$ are independent Gaussian random variables whose dispersion is unity and

$$
0<a_{0}, \quad 1=\left|a_{1}\right|>\left|a_{2}\right|>\cdots>\left|a_{4 N}\right| .
$$

Its average and dispersion are

$$
\left\langle\delta_{r}\right\rangle=a_{0} \sum_{i=1}^{2 N} a_{i},
$$




$$
\sigma^{2}=\left\langle\delta_{r}^{2}\right\rangle-\left\langle\delta_{r}\right\rangle^{2}=a_{0}^{2}\left(\sum_{i=1}^{2 N} a_{i}^{2}\left\langle x_{i}^{4}\right\rangle+\sum_{i \neq j} a_{i} a_{j}-\sum_{i=1}^{2 N} a_{i}^{2}-\sum_{i \neq j} a_{i} a_{j}\right)=2 a_{0}^{2} \sum_{i=1}^{2 N} a_{i}^{2} .
$$

These can also be calculated from Eqs. (4.6) and (4.7). For $\eta=\left(0.7 k_{p}\right)^{-1},\left\langle\delta_{r}\right\rangle \simeq-0.69$ and $\sigma \simeq 1.03$, and these values have also been obtained in the numerical computations (see (5.68) below), which serves as a crosscheck. We chose $\epsilon=0.05$ and $d k=\epsilon k_{p}$. In this case, $N$ turns out to be 2517 , but interestingly more than $95 \%$ of $\delta_{r}$ is determined by only the first 24 terms with the rest negligible, namely,

$$
\frac{\sum_{i=1}^{24}\left|a_{i}\right|}{\sum_{i=1}^{2 N}\left|a_{i}\right|} \simeq 0.98
$$

Consequently, in deriving the PDF of $\delta_{r}$ one can focus only on them, safely neglecting the rest, which greatly simplifies the analysis. We found

$$
\begin{aligned}
& \left\langle\delta_{r}\right\rangle \simeq-0.69, \sigma \simeq 1.0, a_{0} \simeq 0.30, a_{1-5} \simeq-1.0, \\
& a_{6-10} \simeq 0.46, a_{11-17} \simeq 0.11, a_{18-24} \simeq-0.078 .
\end{aligned}
$$

We have also calculated the coefficients for $\epsilon=0.1, d k=\epsilon k_{p}$ and also for $\epsilon=0.1, d k=2 \epsilon k_{p} / 3$, and the results coincided with the above well. Hence we can conclude that the above choices of $\epsilon=0.05$ and $d k=\epsilon k_{p}$ are sufficiently small to obtain reliable results.

We are in a position to discuss the PDF of $\delta_{r}$ using the coefficients of (5.68). First one can resort to a brute-force method of a Monte Carlo simulation to obtain the PDF of $\delta_{r}$, by simply generating 24 random Gaussian variables with dispersion unity, $x_{1}, x_{2}, \cdots, x_{24}$, and summing up the square of them with the coefficients above. We have generated $\left\{x_{i}\right\}$ a million times to obtain the PDF of $\delta_{r}$, shown in Fig. 3. In this appendix $\mathcal{A}$ is set to unity, and so what is shown there is the PDF of $\tilde{\delta_{r}} \equiv\left(\delta_{r}-\left\langle\delta_{r}\right\rangle\right) / \mathcal{A}^{2}$.

We adopt the Clopper-Pearson interval [83] to obtain the $95 \%$ confidence interval $p_{L}<p<p_{U}$ of the probability $p$ of $\delta_{r}$ being realized in some interval $\left(\delta_{r} \pm d \delta_{r}\right)$, when $\delta_{r}$ in that range is realized $k$ times in $N$ trials, as follows. First, the number of an event with probability $p$ realized in $N$ trials follows a Binomial distribution: $P(k ; p)={ }_{N} C_{k} p^{k}(1-p)^{N-k}$. Let us introduce $\alpha=1-C, C=0.95$. From the meaning of the confidence interval, the probability of the event being realized less than $k$ times when $p=p_{U}$ is $\alpha / 2$ :

$$
\sum_{i=0}^{k} P\left(i ; p_{U}\right)=I\left(1-p_{U}, N-k, 1+k\right)=1-I\left(p_{U}, 1+k, N-k\right)=\frac{\alpha}{2},
$$

where $I(x, a, b)$ is the regularized beta function and the relation $I(x, a, b)=I(1-x, b, a)$ has been used. From this, $p_{U}$ can be expressed by the inverse $I^{-1}$ of the regularized beta function as

$$
p_{U}=I^{-1}\left(1-\frac{\alpha}{2}, 1+k, N-k\right) .
$$

Similarly, the probability of the event being realized more than $k$ times when $p=p_{L}$ is $\alpha / 2$ :

$$
\sum_{i=k}^{N} P\left(i ; p_{L}\right)=1-I\left(1-p_{L}, N-k+1, k\right)=I\left(p_{L}, k, N-k+1\right)=\frac{\alpha}{2},
$$

which leads to

$$
p_{L}=I^{-1}\left(\frac{\alpha}{2}, k, N-k+1\right) .
$$

The error bars in Fig. 3 are obtained from (5.70) and (5.72). 
Finally let us discuss an approximate formula for the PDF. Noting that the first ten terms of (5.63) give dominant contributions, we begin by deriving the PDF of $Z=-X+c Y$, where $X$ and $Y$ are both random variables following the chi-squared distribution with $n$ degrees of freedom and $c$ is a positive constant. The PDF of both $\mathrm{X}$ and $\mathrm{Y}$ is

$$
P_{1}(n ; X)=\frac{(1 / 2)^{n / 2}}{\Gamma(n / 2)} X^{n / 2-1} e^{-X / 2} .
$$

Then the PDF of $Z$ is

$$
\begin{aligned}
P_{2}(n, c ; Z) & =N_{1} \int_{0}^{\infty} d X \int_{0}^{\infty} d Y \delta(Z+X-c Y) P_{1}(n ; X) P_{1}(n ; Y) \\
& =\frac{N_{1}(1 / 2)^{n}}{\Gamma(n / 2)^{2}} e^{-\frac{Z}{2 c}}\left(\frac{1}{c}\right)^{\frac{n}{2}-1} \int_{\max \{0,-Z\}}^{\infty} d X X^{\frac{n}{2}-1} e^{-\frac{X}{2}}(Z+X)^{\frac{n}{2}-1} e^{-\frac{X}{2 c}} \\
& =\frac{N_{1}}{\sqrt{2 \pi 2^{n}} \Gamma(n / 2)} c^{1-n / 2} \exp \left(-\frac{1-c}{4 c} Z\right)\left(\frac{c|Z|}{1+c}\right)^{(n-1) / 2} K_{(n-1) / 2}\left(\frac{1+c}{4 c}|Z|\right),
\end{aligned}
$$

where $N_{1}$ is a normalization factor and $K_{m}(x)$ is the modified Bessel function of second kind. In deriving the PDF of $\delta_{r}$, one may simply replace the terms $11 \leq i$ in (5.63) by their expectation values $E \equiv 7 a_{11}+7 a_{18}$ since they are relatively unimportant, and then finally

$$
P\left(\tilde{\delta}_{r}\right) \simeq P_{2}\left(5, a_{6} ; \frac{\tilde{\delta}_{r}+\left\langle\delta_{r}\right\rangle / \mathcal{A}^{2}}{a_{0}}-E\right) .
$$

Interestingly, this approximates the PDF inferred from the Monte Carlo simulation mentioned above overall fairly well, as is shown in Fig. 3. In more detail, this formula slightly deviates from the simulated points around $\tilde{\delta}_{r} \sim 0$, presumably because the terms $11 \leq i$, simply replaced by their expectation values to obtain the above approximate formula, are relatively important there. On the other hand, this formula is better for $\left|\tilde{\delta}_{r}\right| \gtrsim 2$, which is probably because the probability of these relatively rare events is mostly determined by the first ten terms, with the rest of the terms lying around their expectation values. Since the probability of PBH formation has to be extremely rare, what matters is only the tail of the PDF, and therefore we can safely use the above approximate formula to calculate the PBHs' abundance and place upper bounds on tensor perturbations from their absences.

\section{ACKNOWLEDGMENTS}

We are grateful to Jun'ichi Yokoyama for reading the manuscript, useful comments and continuous encouragement. We also thank Kazunari Eda, Yuki Watanabe, Yosuke Itoh, Daisuke Yamauchi, Tsutomu Kobayashi, Masahide Yamaguchi, Takahiro Tanaka for helpful comments. This work was partially supported by Grant-in-Aid for JSPS Fellow No. 25.8199 (T.N.), JSPS Postdoctoral Fellowships for Research Abroad (T.N.), JSPS Grant-in-Aid for Young Scientists (B) No. 15K17632 (T.S.), MEXT Grant-in-Aid for Scientific Research on Innovative Areas "New Developments in Astrophysics Through Multi-Messenger Observations of Gravitational Wave Sources" No. 15H00777 (T.S.) and "Cosmic Acceleration" No. $15 \mathrm{H} 05888$ (T.S.)

[1] Planck Collaboration, P. Ade et al., Astron.Astrophys. 571, A16 (2014), 1303.5076. 
[2] BICEP2 Collaboration, P. Ade et al., Phys.Rev.Lett. 112, 241101 (2014), 1403.3985.

[3] B. Allen, (1996), gr-qc/9604033.

[4] T. L. Smith, E. Pierpaoli, and M. Kamionkowski, Phys.Rev.Lett. 97, 021301 (2006), astro$\mathrm{ph} / 0603144$.

[5] Y. Kikuta, K. Kohri, and E. So, (2014), 1405.4166.

[6] A. Ota, T. Takahashi, H. Tashiro, and M. Yamaguchi, (2014), 1406.0451.

[7] J. Chluba, L. Dai, D. Grin, M. Amin, and M. Kamionkowski, Mon.Not.Roy.Astron.Soc. 446, 2871 (2015), 1407.3653.

[8] G. Mangano et al., Nucl. Phys. B729, 221 (2005), hep-ph/0506164.

[9] K. Ichiki, M. Yahiro, T. Kajino, M. Orito, and G. Mathews, Phys.Rev. D66, 043521 (2002), astro-ph/0203272.

[10] P. S. Apostolopoulos and N. Tetradis, Phys.Lett. B633, 409 (2006), hep-th/0509182.

[11] R. Maartens and K. Koyama, Living Rev.Rel. 13, 5 (2010), 1004.3962.

[12] T. Nakama and T. Suyama, Phys. Rev. D92, 121304 (2015), 1506.05228.

[13] Y. B. Zel'dovich and I. D. Novikov, Sov. Astron. 10, 602 (1967).

[14] B. J. Carr and S. Hawking, Mon.Not.Roy.Astron.Soc. 168, 399 (1974).

[15] B. J. Carr, Astrophys.J. 201, 1 (1975).

[16] T. Harada, C.-M. Yoo, and K. Kohri, Phys.Rev. D88, 084051 (2013), 1309.4201.

[17] D. K. Nadezhin, I. D. Novikov, and A. G. Polnarev, Sov. Astron. 22, 129 (1978).

[18] M. Shibata and M. Sasaki, Phys.Rev. D60, 084002 (1999), gr-qc/9905064.

[19] J. C. Niemeyer and K. Jedamzik, Phys.Rev. D59, 124013 (1999), astro-ph/9901292.

[20] A. G. Polnarev and I. Musco, Class.Quant.Grav. 24, 1405 (2007), gr-qc/0605122.

[21] A. Polnarev, T. Nakama, and J. Yokoyama, JCAP 1209, 027 (2012), 1204.6601.

[22] T. Nakama, T. Harada, A. Polnarev, and J. Yokoyama, JCAP 1401, 037 (2014), 1310.3007.

[23] T. Nakama, JCAP 1410, 040 (2014), 1408.0955.

[24] B. Carr, K. Kohri, Y. Sendouda, and J. Yokoyama, Phys.Rev. D81, 104019 (2010), 0912.5297.

[25] E. Bugaev and P. Klimai, Phys.Rev. D79, 103511 (2009), 0812.4247.

[26] A. S. Josan, A. M. Green, and K. A. Malik, Phys.Rev. D79, 103520 (2009), 0903.3184.

[27] J. Barrow and P. Coles, Mon. Not. Roy. Astron. Soc. 248, 52 (1991).

[28] J. Chluba and R. Sunyaev, (2011), 1109.6552.

[29] J. Chluba, A. L. Erickcek, and I. Ben-Dayan, Astrophys.J. 758, 76 (2012), 1203.2681.

[30] J. Chluba, R. Khatri, and R. A. Sunyaev, (2012), 1202.0057.

[31] J. B. Dent, D. A. Easson, and H. Tashiro, Phys.Rev. D86, 023514 (2012), 1202.6066.

[32] R. Khatri and R. A. Sunyaev, JCAP 1209, 016 (2012), 1207.6654.

[33] R. A. Sunyaev and R. Khatri, Int.J.Mod.Phys. D22, 1330014 (2013), 1302.6553.

[34] R. Khatri and R. A. Sunyaev, JCAP 1306, 026 (2013), 1303.7212.

[35] J. Chluba and D. Grin, Mon.Not.Roy.Astron.Soc. 434, 1619 (2013), 1304.4596.

[36] J. Chluba and D. Jeong, (2013), 1306.5751.

[37] T. Nakama, T. Suyama, and J. Yokoyama, Phys.Rev.Lett. 113, 061302 (2014), 1403.5407.

[38] D. Jeong, J. Pradler, J. Chluba, and M. Kamionkowski, Phys.Rev.Lett. 113, 061301 (2014), 1403.3697 .

[39] T. Bringmann, P. Scott, and Y. Akrami, Phys.Rev. D85, 125027 (2012), 1110.2484.

[40] K. Kohri, T. Nakama, and T. Suyama, Phys.Rev. D90, 083514 (2014), 1405.5999.

[41] S. Matarrese, O. Pantano, and D. Saez, Phys.Rev.Lett. 72, 320 (1994), astro-ph/9310036.

[42] S. Matarrese, S. Mollerach, and M. Bruni, Phys.Rev. D58, 043504 (1998), astro-ph/9707278.

[43] C. Carbone and S. Matarrese, Phys.Rev. D71, 043508 (2005), astro-ph/0407611. 
[44] K. N. Ananda, C. Clarkson, and D. Wands, Phys.Rev. D75, 123518 (2007), gr-qc/0612013.

[45] D. Baumann, P. J. Steinhardt, K. Takahashi, and K. Ichiki, Phys.Rev. D76, 084019 (2007), hep-th/0703290.

[46] L. Alabidi, K. Kohri, M. Sasaki, and Y. Sendouda, JCAP 1209, 017 (2012), 1203.4663.

[47] E. Bugaev and P. Klimai, Phys.Rev. D83, 083521 (2011), 1012.4697.

[48] R. Saito and J. Yokoyama, Phys.Rev.Lett. 102, 161101 (2009), 0812.4339, [107, 069901(E) (2011).].

[49] R. Saito and J. Yokoyama, Prog.Theor.Phys. 123, 867 (2010), 0912.5317, [126, 351(E) (2011).].

[50] E. Bugaev and P. Klimai, Phys.Rev. D81, 023517 (2010), 0908.0664.

[51] D. R. Brill, Annals Phys. 7, 466 (1959).

[52] K. Eppley, Phys.Rev. D16, 1609 (1977).

[53] S. M. Miyama, Prog.Theor.Phys. 65, 894 (1981).

[54] M. Shibata, K.-i. Nakao, T. Nakamura, and K.-i. Maeda, Phys.Rev. D50, 708 (1994).

[55] M. Shibata and T. Nakamura, Phys.Rev. D52, 5428 (1995).

[56] P. Anninos, J. Masso, E. Seidel, W.-m. Suen, and M. Tobias, Phys.Rev. D54, 6544 (1996), gr-qc/9601026.

[57] M. Shibata, Phys.Rev. D55, 7529 (1997).

[58] M. Alcubierre et al., Phys.Rev. D62, 044034 (2000), gr-qc/0003071.

[59] H. P. Pfeiffer, L. E. Kidder, M. A. Scheel, and D. Shoemaker, Phys.Rev. D71, 024020 (2005), gr-qc/0410016.

[60] L. A. Boyle, P. J. Steinhardt, and N. Turok, Phys. Rev. D69, 127302 (2004), hep-th/0307170.

[61] M. Baldi, F. Finelli, and S. Matarrese, Phys. Rev. D72, 083504 (2005), astro-ph/0505552.

[62] E. J. Copeland, D. J. Mulryne, N. J. Nunes, and M. Shaeri, Phys. Rev. D79, 023508 (2009), 0810.0104.

[63] T. Kobayashi, M. Yamaguchi, and J. Yokoyama, Prog. Theor. Phys. 126, 511 (2011), 1105.5723.

[64] T. Biswas, T. Koivisto, and A. Mazumdar, JHEP 08, 116 (2014), 1403.7163.

[65] A. Ashoorioon, K. Dimopoulos, M. M. Sheikh-Jabbari, and G. Shiu, Phys. Lett. B737, 98 (2014), 1403.6099.

[66] D. Cannone, G. Tasinato, and D. Wands, JCAP 1501, 029 (2015), 1409.6568.

[67] L. Graef and R. Brandenberger, JCAP 1510, 009 (2015), 1506.00896.

[68] S. Weinberg, Oxford University Press (2008).

[69] D. Baumann, 0907.5424.

[70] M. Maggiore, Oxford University Press (2008).

[71] M. Maggiore, Phys.Rept. 331, 283 (2000), gr-qc/9909001.

[72] S. Kuroyanagi, T. Takahashi, and S. Yokoyama, (2014), 1407.4785.

[73] I. Sendra and T. L. Smith, Phys. Rev. D85, 123002 (2012), 1203.4232.

[74] LIGO Scientific, VIRGO, J. Aasi et al., Phys.Rev.Lett. 113, 231101 (2014), 1406.4556.

[75] A. Nishizawa and N. Seto, Phys.Rev. D91, 122001 (2015), 1503.00354.

[76] J. H. MacGibbon, Nature 329, 308 (1987).

[77] A. D. Dolgov and D. Ejlli, Phys. Rev. D84, 024028 (2011), 1105.2303.

[78] T. Harada, C.-M. Yoo, T. Nakama, and Y. Koga, Phys. Rev. D91, 084057 (2015), 1503.03934.

[79] B. J. Carr and J. E. Lidsey, Phys. Rev. D48, 543 (1993).

[80] C. T. Byrnes, E. J. Copeland, and A. M. Green, Phys. Rev. D86, 043512 (2012), 1206.4188.

[81] T. Nakama and T. Suyama, in prep. (2016). 
[82] C. Pitrou, X. Roy, and O. Umeh, Class.Quant.Grav. 30, 165002 (2013), 1302.6174.

[83] C. Clopper and E. S. Pearson, Biometrika 26, 404 (1934). 